\title{
A scale and aerosol aware stochastic convective parameterization for weather and air quality modeling
}

\author{
G. A. Grell ${ }^{1}$ and S. R. Freitas ${ }^{2}$ \\ ${ }^{1}$ Earth Systems Research Laboratory of the National Oceanic and Atmospheric Administration (NOAA), Boulder, \\ Colorado 80305-3337, USA \\ ${ }^{2}$ Center for Weather Forecasting and Climate Studies, INPE, Cachoeira Paulista, Sao Paulo, Brazil \\ Correspondence to: G. A. Grell (georg.a.grell@ noaa.gov)
}

Received: 27 July 2013 - Published in Atmos. Chem. Phys. Discuss.: 11 September 2013

Revised: 30 January 2014 - Accepted: 25 March 2014 - Published: 27 May 2014

\begin{abstract}
A convective parameterization is described and evaluated that may be used in high resolution non-hydrostatic mesoscale models as well as in modeling system with unstructured varying grid resolutions and for convection aware simulations. This scheme is based on a stochastic approach originally implemented by Grell and Devenyi (2002). Two approaches are tested on resolutions ranging from $20 \mathrm{~km}$ to $5 \mathrm{~km}$. One approach is based on spreading subsidence to neighboring grid points, the other one on a recently introduced method by Arakawa et al. (2011). Results from model intercomparisons, as well as verification with observations indicate that both the spreading of the subsidence and Arakawa's approach work well for the highest resolution runs. Because of its simplicity and its capability for an automatic smooth transition as the resolution is increased, Arakawa's approach may be preferred. Additionally, interactions with aerosols have been implemented through a cloud condensation nuclei $(\mathrm{CCN})$ dependent autoconversion of cloud water to rain as well as an aerosol dependent evaporation of cloud drops. Initial tests with this newly implemented aerosol approach show plausible results with a decrease in predicted precipitation in some areas, caused by the changed autoconversion mechanism. This change also causes a significant increase of cloud water and ice detrainment near the cloud tops. Some areas also experience an increase of precipitation, most likely caused by strengthened downdrafts.
\end{abstract}

\section{Introduction}

There are many different parameterizations for deep and shallow convection that exploit the current understanding of the complicated physics and dynamics of convective clouds to express the interaction between the larger scale flow and the convective clouds in simple "parameterized" terms. These parameterizations often differ fundamentally in closure assumptions and parameters used to solve the interaction problem, leading to a large spread and uncertainty in possible solutions. For some interesting review articles on convective parameterizations the reader is referred to Frank (1984), Grell (1991), Emanuel and Raymond (1992), Emanuel (1994), and Arakawa (2004). New ideas that have recently been implemented include built-in stochasticism (Grell and Devenyi, 2002; Lin and Neelin, 2003), the super parameterization approach (Grabowski and Smolarkiewicz, 1999; Randall et al., 2003), and a lattice type stochastic multi-cloud model for convective parameterizations (Khouider 2014).

An additional complication that is gaining attention rapidly is the use of convective parameterizations on so called "gray scales" (Kuell et al., 2007; Mironov, 2009; Gerard et al., 2009; Yano et al., 2010). With the increase in computer power, high resolution numerical modeling using horizontal grid scales of $d x<10 \mathrm{~km}$ is becoming widespread, even at operational centers. On these types of resolutions, many of the assumptions that are made in deriving the theory behind convective parameterizations are no longer valid. On the other hand, to properly resolve convection, the horizontal resolutions of these gray scales are also inadequate (see also 
Bryan et al., 2003; Hong and Dudhia, 2012). Optimally, a convective parameterization should be scale dependent (see also Arakawa et al., 2011) with assumptions that may vary with horizontal resolution.

Yet another complicating factor is the increased development of integrated models that combine weather and chemistry. Until recently, because of the complexity and the lack of appropriate computer power, air chemistry and weather modeling have developed as separate disciplines, leading to the development of separate modeling systems that were only loosely coupled. It is well accepted that weather is of decisive importance for air quality, or for the aerial transport of hazardous materials. It is also recognized that chemical species will influence the weather by changing the atmospheric radiation budget as well as through cloud formation. While many of these coupled modeling systems include sub-grid scale transport of chemical constituents and interaction of aerosols with radiation as well as interaction with microphysical schemes for explicit treatment of the aerosol indirect effect, little work has been done trying to couple aerosols with convective parameterizations.

In this paper we discuss the development of a convective parameterization that addresses the gray scale issue, transport of chemical constituents, and possible interactions with aerosols. In Sect. 2 of this paper we will briefly discuss the issues involved when parameterizing convection on gray scales. Section 3 will discuss the main aspects of our convective parameterization as it is applied in numerical weather prediction models, including the transport of tracers, and interactions with aerosols. Section 4 will show some results, and Sect. 5 will give conclusions. The parameterization that we describe below has been released to users of the Weather Research and Forecasting (WRF, Skamarock et al., 2008) modeling system as well as the Brazilian version of the Regional Atmospheric Modeling system (BRAMS, Freitas et al., 2009).

\section{Parameterizing convection on "almost" cloud resolving scales}

Although the purpose of this paper is not to give a review of the problem and discuss attempts to solve it, we will give an abbreviated overview of different approaches that may be implemented in our parameterization. The need for parameterizations arises from the existence of important processes (processes that influence the explicitly describable scales in the model) that are occurring on scales too small to be accurately resolved (or resolved at all) explicitly. The hypothesis in parameterizations (at least of convection) is that the effects of these unresolvable scales are describable (at least to some acceptable level of uncertainty) in terms of the state and history of the explicitly described scales.

Traditionally, parameterizations of convection have been designed to be self-contained within one grid column, under the assumption that the fraction of the grid column that is occupied by active convection is small. However, this assumption starts to break down as horizontal grid spacing diminishes. An adverse result in model simulations, where the user may have the need to resolve some of the convection (such as may be possible for fronts, and mesoscale convective complexes (MCCs) or systems (MCSs)), may be that the strong subgrid-scale subsidence effects may inhibit the model from explicitly resolving any part of the convective system. The flow with respect to convection becomes more viscous. $\mathrm{Nu}-$ merically, with increasing horizontal resolution (in particular with $d x<10 \mathrm{~km}$ ), the heating and drying caused by compensating subsidence within one grid box may inhibit the explicit microphysical parameterizations. The degree of inhibition depends on the strength of the subsidence versus the resolved scale vertical ascent. However, explicit treatment of some of these mesoscale systems is essential for a much more realistic simulation of the physical processes involved.

Since even operational centers are applying horizontal resolutions much finer than $20 \mathrm{~km}$, several approaches have recently been discussed to address some of the scale separation issues. In this paper we will focus on two ideas that may be used in our parameterization. We are excluding the super-parameterization approach (or targeted nesting, where a cloud model may be nested within itself, Grabowski and Smolarkiewicz, 1999; Randall et al., 2003) since it is not based on a convective parameterization, but recognize that with increasing computing power it may be promising in future applications. We are also excluding other recently discussed approaches that cannot easily be employed in our parameterization (Yano et al., 2010; Gerard et al., 2009; Mironov, 2009)

The two ideas discussed here stem from either a look at a more theoretical approach (Arakawa et al. (2011), hereafter A2011), where the equations for the eddy fluxes are re-derived to introduce a dependence on the fractional area coverage, or they are based more explicitly on a simple conceptual picture of a convective cloud (Fig. 1), relaxing the assumption that the eddy fluxes are within one grid box. A2011 first re-derive the Reynolds averaged equations for the vertical eddy flux terms. In short, letting the overbar denote a grid box average, the tilde represents the environmental component, subscript $\mathrm{c}$ indicates the convective portion of variable $\psi$, and let $\sigma$ be the fractional area coverage of convection, then

$\bar{\psi}=\sigma \bar{\psi}_{\mathrm{c}}+(1-\sigma) \tilde{\psi}$,

$\bar{w}=\sigma \bar{w}_{\mathrm{c}}+(1-\sigma) \tilde{w}$,

$\overline{w \psi}=\sigma{\overline{w_{\mathrm{c}}}}_{\mathrm{c}}+(1-\sigma) \tilde{w \psi}$,

therefore

$\overline{w \psi}-\bar{w} \bar{\psi}=\frac{\sigma}{1-\sigma}\left(w_{\mathrm{c}}-\bar{w}\right)\left(\psi_{\mathrm{c}}-\bar{\psi}\right)$, 


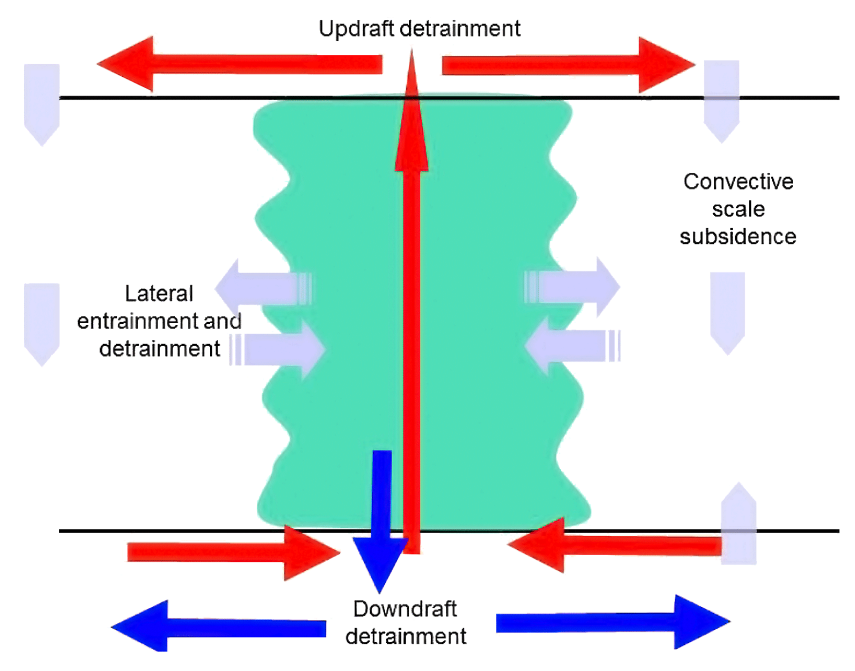

Figure 1. Conceptual picture of a convective cloud.

and with $\sigma \ll 1$ and $w_{\mathrm{c}} \gg \tilde{w}$,

$\rho(\overline{w \psi}-\bar{w} \bar{\psi}) \approx m_{\mathrm{c}}\left(\psi_{\mathrm{c}}-\bar{\psi}\right)=\sigma \rho w_{\mathrm{c}}\left(\psi_{\mathrm{c}}-\bar{\psi}\right)$.

Here $m_{\mathrm{c}}$ is the convective mass flux. Equation (5) is commonly used by mass flux type schemes to parameterize deep convection. This simple equation can easily be related to a conceptual picture of a convective cloud, shown in Fig. 1, but is somewhat more general since it is not restricted to just one particular cloud type.

It is obvious that the assumption $\sigma \ll 1$ breaks down as the horizontal resolution is increased. A unified approach for convective parameterizations is introduced by A2011, which re-derive the vertical eddy fluxes by assuming that since the parameterization must converge to an explicit simulation of cloud processes as $\sigma \rightarrow 1$, it follows that $\lim w_{\mathrm{c}}=\bar{w}$ and $\lim \psi_{\mathrm{c}}=\bar{\psi}$. They continue by simply defining a choice that satisfies that requirement and end up with the simple equation

$\overline{w \psi}-\bar{w} \bar{\psi}=(1-\sigma)^{2}(\overline{w \psi}-\bar{w} \bar{\psi})_{\mathrm{adj}}$,

where $(\overline{w \psi}-\bar{w} \bar{\psi})_{\text {adj }}$ is the solution when $\sigma \ll 1$. Assuming an appropriate parameterization for $\sigma$ can be found (see also A2011), Eq. (6) is a very simple option that may also be used in our parameterization.

The other approach is based on interpreting the conceptual picture in Fig. 1, where the cloud is envisioned as the statistical average of a deep convective cloud that may occur at that grid cell, given the environmental conditions at that time. Given a closure to determine the mass flux and/or fractional area coverage and updraft or downdraft vertical velocity the vertical eddy fluxes due to unresolved convection are then usually determined through lateral mixing, convective scale compensating subsidence (or uplifting caused by downdraft mass flux), and "massive" detrainment at the cloud top or downdraft bottom. By far the largest effects happen through compensating subsidence (usually a strong heating and drying effect), and the massive detrainments from updrafts and downdrafts. Physically, with increasing resolution and Fig. 1 in mind, compensating subsidence, as well as massive entrainment and detrainment at the cloud bottom and top, may be spread over larger areas than a single grid box. A simple idea that has been used in our parameterization within WRF and BRAMS is to assume that we are not looking at just one grid cell, but also the nearest neighbor grid cells, and simply distributing massive entrainment, detrainment, and subsidence over the neighboring grid cells. This approach (termed G3d, based on Grell and Devenyi, 2002) will be compared to Arakawa's approach and evaluated with observations.

Finally, there exists an interesting third approach that may be used in our parameterization. It was introduced by Kuell et al. (2007) and is applicable only for non-hydrostatic models, by letting the parameterization only transport mass, assuming that the model will then handle the subsidence. From Fig. 1 this will still assume that the massive detrainment is in one grid box, but the subsidence heating and drying is left for the model to do. Kuell et al. (2007) show nice results when applied within the NWP model of the German Weather Service. This idea can be used in other non-hydrostatic cloud resolving models and may also be implemented in our modeling systems with our parameterization. However, implementation is not as straight forward as Arakawa's approach and we refrained from testing this method in this paper.

\section{The convective parameterization}

The parameterization framework is a simple scheme that is based on a convective parameterization developed by Grell (1993, G1) and expanded by Grell and Devenyi (2002, GD) to include stochasticism. In short, the scheme described in G1 was expanded to allow for a series of different assumptions that are commonly used in convective parameterizations and that have proven to lead to large sensitivity in model simulations. In addition, values for the assumed parameters may be perturbed using random number generators. We refer the reader to G1 and GD for numerical details of the scheme, but we will describe differences as they exist in the current version. The GD scheme can use a very large number of ensemble members, but in operational applications this number has to be restricted because of computing time requirements. It is therefore important to choose ensembles that will give the biggest "bang for the buck". GD was modified later (G3d) to include options to spread subsidence to neighboring grid points. An application of the ensemble version using Bayesian data assimilation is described in GD. Another interesting approach that makes use of the stochasticism is presented in Santos et al. (2013), who use a statistical method to increase the forecast skill for precipitation. The basic G3d parameterization is currently used in research and forecasting 

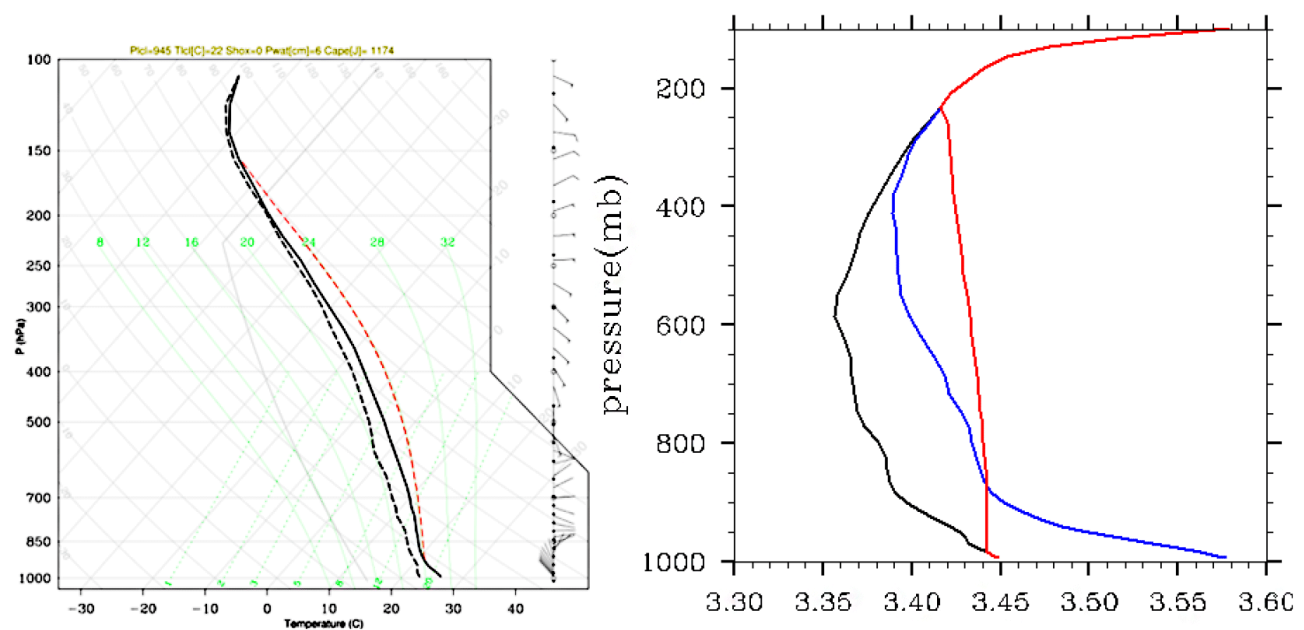

Figure 2. Skewt diagram (left) displaying temperature (solid black line), dew point (dashed black line), vertical wind profile and the CAPE (Convective Available Potential Energy) parcel profile (dashed red). Also shown are vertical profiles (right) of moist static energy (black), saturation moist static energy (blue) and simulated updraft moist static energy (red). Units for the abscissa.

applications using the WRF model, the BRAMS system, and in an operational application in the Rapid Refresh System (RAP, http://rapidrefresh.noaa.gov). Currently all ice phase processes are still neglected.

\subsection{The basic ensemble equations}

Following GD, the non-resolved fluxes from convective clouds are described by

$$
\begin{aligned}
& \left(\frac{\partial s}{\partial t}\right)_{\mathrm{c}} \equiv \overline{\left(\frac{\partial s}{\partial t}\right)_{\mathrm{c}}} \equiv-\frac{1}{\rho} \frac{\partial}{\partial z}\left(\overline{F_{\mathrm{s}}}-L \overline{F_{1}}\right) \\
& \left(\frac{\partial q}{\partial t}\right)_{\mathrm{c}} \equiv \overline{\left(\frac{\partial q}{\partial t}\right)_{\mathrm{c}}} \equiv-\frac{1}{\rho} \frac{\partial}{\partial z}\left(\bar{F}_{q}+\overline{F_{1}}\right)-\bar{R}
\end{aligned}
$$

where $s$ is the dry static energy $\left(s=c_{\mathrm{p}} T+g z\right), q$ is the water vapor mixing ratio, and $\rho$ is the density. $\overline{F_{\mathrm{S}}}$ is the ensemble averaged flux of dry static energy, $\overline{F_{q}}$ is the ensemble averaged flux of water vapor, $\overline{F_{1}}$ is the ensemble averaged flux of suspended cloud liquid water, $L$ is the latent heat of vaporization and $\bar{R}$ is the ensemble averaged convective precipitation. While for Eqs. (1)-(6), the overbar referred to Reynolds averaging, for Eqs. (7) and (8), and all subsequent equations the overbar will denote an ensemble average. The ensemble average of $N$ un-weighted ensemble members is simply defined as

$\bar{X}=\frac{1}{N} \sum_{n=1}^{n=N} x^{n}$
The fluxes for ensemble member $n$ are defined as

$$
\begin{aligned}
& F_{\mathrm{s}}^{n}(z)= \\
& {\left[s_{\mathrm{u}}^{n}(z)-\tilde{s}(z)\right] m_{\mathrm{u}}^{n}(z)-\left[s_{\mathrm{d}}^{n}(z)-\tilde{s}(z)\right] m_{\mathrm{d}}^{n}(z),} \\
& F_{q}^{n}(z)= \\
& {\left[q_{\mathrm{u}}^{n}(z)-\tilde{q}(z)\right] m_{\mathrm{u}}^{n}(z)-\left[q_{\mathrm{d}}^{n}(z)-\tilde{q}(z)\right] m_{\mathrm{d}}^{n}(z),}
\end{aligned}
$$

and

$F_{1}^{n}(z)=l^{n}(z) m_{\mathrm{u}}^{n}(z)$.

The subscript $u$ refers to the updraft, and the subscript $\mathrm{d}$ to the downdraft. The tildes indicate a mean, environmental value. The quantity $l(z)$ is the suspended mixing ratio of liquid water. The mass flux $m$ is then normalized by the mass flux at cloud base $m_{\mathrm{b}}$ to give

$m_{\mathrm{u}}^{n}(z)=m_{\mathrm{b}}^{n} \eta_{\mathrm{u}}^{n}(z)$

and

$m_{\mathrm{d}}^{n}(z)=\epsilon m_{\mathrm{b}}^{n} \eta_{\mathrm{d}}^{n}(z)$.

Since our parameterization is used for operational applications, computational efficiency is essential. To accomplish this, several simplifications are made for the above ensemble equations. GD use a variety of closures to calculate $m_{\mathrm{b}}^{n}$. Within the framework that was described in G1 and used in GD, implementing these closures is an easy task and requires almost no additional computational resources. While in GD as well as G3d $m_{\mathrm{b}}^{n}$ is not independent of other ensembles, this is not the case in the Grell and Freitas (GF) parameterization described here, since the number of ensembles has been reduced significantly for more efficient operational applications. We therefore treat the calculation of the mass fluxes 
separately by first assuming that

$\overline{m_{\mathrm{b}}}=\frac{1}{N} \sum_{n=1}^{n=N} m_{\mathrm{b}}^{n}$

and then substituting Eq. (15) into Eqs. (10)-(14). To give one example, the equations for the fluxes then become

$F_{\mathrm{S}}^{n}(z)=$

$\overline{m_{\mathrm{b}}}\left\{\left[s_{\mathrm{u}}^{n}(z)-\tilde{s}(z)\right] \eta_{\mathrm{u}}^{n}(z)-\left[s_{\mathrm{d}}^{n}(z)-\tilde{s}(z)\right] \eta_{\mathrm{d}}^{n}(z)\right\}$,

$F_{q}^{n}(z)=$

$\overline{m_{\mathrm{b}}}\left\{\left[q_{\mathrm{u}}^{n}(z)-\tilde{q}(z)\right] \eta_{\mathrm{u}}^{n}(z)-\left[q_{\mathrm{d}}^{n}(z)-\tilde{q}(z)\right] \eta_{\mathrm{d}}^{n}(z)\right\}$,

$F_{1}^{n}(z)=\overline{m_{\mathrm{b}}} l^{n}(z) \eta_{\mathrm{u}}^{n}(z)$

Since $\overline{m_{\mathrm{b}}}$ does not depend on $z$ and is already an ensemble average (essentially it becomes a constant), Eqs. (7) and (8) then only depend linearly on $\overline{m_{\mathrm{b}}}$, as well as the normalized fluxes defined in Eqs. (16)-(18). The normalized fluxes are dependent on the simple cloud model that is chosen, as well as possible perturbations on some of the assumptions that are used. All numerical approximations are as in G1, except for the modifications described as follows. The calculation of $\overline{m_{\mathrm{b}}}$ is very simple and depends upon the choices of trigger functions and closure assumptions (including perturbations of the closures). Additionally, observed rainfall rates $(R)$ may also be used to determine this variable. This may be useful for data assimilation purposes. In this case, following GD we get

$\overline{m_{\mathrm{b}}}=\frac{R}{\overline{I_{1}}(1-\bar{\beta})}$,

where $1-\bar{\beta}$ is the precipitation efficiency and $I_{1}$ is the normalized condensate.

\subsection{Further modifications in GF compared to GD and G3d}

The normalized mass flux for the updraft, and, separately, the downdraft is usually calculated using

$\epsilon-\delta=\frac{1}{\eta} \frac{\partial \eta}{\partial z}$

Here $\epsilon$ and $\delta$ are the mass entrainment and detrainment (respectively) and simply depend on entrainment and detrainment rates. In GD and G3d we assumed initial conditions at the updraft originating level and downdraft originating level of $\eta=1$. In GF, to get a smoother transition, we assume that the normalized mass flux approaches the value of 1 quadratically from originating level to the level of free convection (for updraft), initially assuming an undiluted ascent. A similar smooth increase is prescribed for downdrafts for the first 5 levels, assuming that the vertical resolution is sufficiently high (otherwise the model will default to the original implementation). To give one example, for the updraft we require a specified normalized mass flux profile from originating level to the level of free convection. We then prescribe a constant detrainment rate (the choice is not important as long as it is small or zero) for these levels and calculate an entrainment rate using Eq. (20) to fulfill the requirement. The entrainment rate at the originating level is an important parameter, since in GF it determines when scale adjustments become important. Its choice is defined and explained in Sect. (4), Eq. (36). Similarly, near the cloud top and downdraft bottom the normalized mass flux profile will go smoothly to zero. For the cloud top, normalized mass flux is assumed to start decreasing when the environment becomes stably stratified. For the downdraft, detrainment is assumed to take place only in the lowest $1000 \mathrm{~m}$ above the ground or starting at the Level of Free Convection (LFC), which ever is located higher above the ground.

To optionally increase diurnal forcing, an excess temperature and moisture perturbation is added when calculating the forcing and checking for trigger functions. This excess value is based on work from Jakob and Siebesma (2003). According to this approach, the boundary condition for temperature and water vapor mixing ratio of the air parcel at initiation level may be modified by adding a perturbation proportional to the surface fluxes, using the following relationships:

$\Delta T=-0.5 \frac{H}{\rho c_{\mathrm{p}} w^{*}}$

and

$\Delta q=-0.5 \frac{L E}{\rho L w^{*}}$

where $H$ and $L E$ are the sensible and latent heat surface fluxes, $\rho$ the air density, $c_{\mathrm{p}}$ is the specific heat at constant pressure for dry air, $L$ the latent heat of evaporation and $w^{*}$ is the convective-scale velocity derived from similarity theory. The factor 0.5 used here was chosen lower than the recommended one $(\sim 1)$ by the authors.

\subsection{Inclusion of tracer transport and wet scavenging}

The modification of a chemical constituent or an inert tracer (C, per unit mass) may be expressed as

$\left(\frac{\partial C}{\partial t}\right)_{\mathrm{c}} \equiv \overline{\left(\frac{\partial C}{\partial t}\right)_{\mathrm{c}}} \equiv-\frac{1}{\rho} \frac{\partial}{\partial z}\left(\overline{F_{\mathrm{c}}}+\overline{F_{\mathrm{lc}}}\right)-\overline{C_{\mathrm{si}}}+\overline{C_{\mathrm{so}}}$,

where subscript sl denotes a sink due to wet deposition, and so denotes a source or sink due to chemical processes. The fluxes are defined as

$$
\begin{aligned}
& F_{\mathrm{c}}^{n}(z)=\overline{m_{\mathrm{b}}}\left\{\left[C_{\mathrm{u}}^{n}(z)-\tilde{C}(z)\right] \eta_{\mathrm{u}}^{n}(z)-\right. \\
& \left.\left[C_{\mathrm{d}}^{n}(z)-\tilde{C}(z)\right] \eta_{\mathrm{d}}^{n}(z)\right\}, p
\end{aligned}
$$

and 
$F_{\mathrm{lc}}^{n}(z)=\overline{m_{\mathrm{b}}} C_{\mathrm{aq}}^{n}(z) \eta_{\mathrm{u}}^{n}(z)$

where $C_{\text {aq }}$ represents the chemical constituent in the aqueous phase. Within WRF-Chem (Grell et al., 2005) a separate routine is used to calculate the fluxes for the chemical species and/or tracers. In order to make this routine available for all other convective parameterizations, $\overline{m_{\mathrm{b}}}$ is recalculated using Eq. (19). In WRF-Chem $C_{\text {so }}$ may be calculated using an aqueous phase chemistry routine. In addition, $\overline{C_{\mathrm{si}}}$ depends on the conversion rate of cloud water to rain water and on the solubility of the tracer. It is calculated using

$$
\frac{\partial}{\partial z} \bar{C}_{\mathrm{si}}=\alpha C m_{\mathrm{u}}^{n} \frac{\partial}{\partial z}\left(q_{\mathrm{r}}^{n}\right)=\alpha \overline{m_{\mathrm{b}}} \eta_{\mathrm{u}}^{n}(z) C \frac{\partial}{\partial z}\left(q_{\mathrm{r}}^{n}\right) .
$$

The variable $\alpha$ can be calculated using Henry's Law, which provides the solubility of the specie in water, or for soluble aerosol constituents, a scavenging constant may be assumed. In WRF-Chem the choice depends somewhat on the chemistry options taken. In general, $\alpha=0.5$, except for sulfate, where $\alpha=1$. For aerosol modules from the GOddard Chemistry Aerosol Radiation and Transport (GOCART) model, the scavenging depends on whether the variable is hydrophobic $(\alpha=0)$ or hydrophilic $(\alpha=0.8)$. Additionally for sea salt, we assume $\alpha=1$. These parameters can easily be changed. It is important to note that wet deposition is one of the most sensitive processes in determining the final concentrations in any model run when precipitation is present.

\subsection{Inclusion of aerosol interactions}

Aerosol interactions are implemented through two processes, conversion of cloud water to rainwater, and evaporation efficiency of rain. In G1, the conversion of cloud water to rainwater is simply dependent on a constant conversion parameter $c_{0}\left(m^{-1}\right)=0.002$. This can simply be derived using a Kessler (1969) approach. Following Kessler, if one neglects the conversion threshold the tendency equation for rainwater $q_{\mathrm{r}}$ (without the ensemble notation), using the Kessler autoconversion rate $k\left(\mathrm{~s}^{-1}\right)$ is

$\frac{d\left(\rho_{\mathrm{c}} q_{\mathrm{r}}\right)}{d t}=w_{\mathrm{c}} \rho_{\mathrm{c}} \frac{\partial}{\partial z}\left(q_{\mathrm{r}}\right)=k \rho_{\mathrm{c}} q_{\mathrm{l}}=m_{\mathrm{u}} \frac{\partial}{\partial z}\left(q_{\mathrm{r}}\right)$,

If we then write the mass budget of the total water vapor $\tilde{q}$ and liquid water $q_{t}$ in an infinitesimal layer of the updraft as

$$
\begin{aligned}
& \frac{\partial}{\partial z}\left(m_{\mathrm{u}}^{n} q_{t}\right)= \\
& \left(\frac{\partial}{\partial z}\left(m_{\mathrm{u}}^{n}\right)\right)_{\varepsilon} \tilde{q}-\left(\frac{\partial}{\partial z}\left(m_{\mathrm{u}}^{n}\right)\right)_{\mathrm{d}} q_{t}^{n}-m_{\mathrm{u}}^{n} \frac{\partial}{\partial z}\left(q_{\mathrm{r}}^{n}\right),
\end{aligned}
$$

and, with the simple autoconversion parameter $c_{0}\left(\mathrm{~m}^{-1}\right)$

$m_{\mathrm{u}}^{n} \frac{\partial}{\partial z}\left(q_{\mathrm{r}}^{n}\right)=c_{0} q_{1}^{n}(z) m_{\mathrm{u}}^{n}=\overline{m_{\mathrm{b}}} \eta_{\mathrm{u}}^{n}(z) \frac{\partial}{\partial z}\left(q_{\mathrm{r}}^{n}\right)$.
Therefore, for the original scheme (G1 and GD), we simply have

$\eta_{\mathrm{u}}^{n}(z) \frac{\partial}{\partial z}\left(q_{\mathrm{r}}^{n}\right)=c_{0} q_{1}^{n}(z) \eta_{\mathrm{u}}^{n}(z)=\frac{k \rho_{\mathrm{c}} q_{1}}{\overline{m_{\mathrm{b}}}}$,

where $q_{1}^{n}$ is the suspended liquid water content in the updraft. From Eq. (30) we can see that $c_{0}$ is chosen assuming an arbitrary base mass flux $\bar{m}_{\mathrm{b}}$ of 0.5 . Optionally, we follow Berry (1968) and parameterize the conversion in terms of cloud condensation nuclei density number $\left(\mathrm{CCN}, \mathrm{cm}^{-3}\right)$ by using

$\overline{m_{\mathrm{b}}} \eta_{\mathrm{u}}^{n}(z) \frac{\partial}{\partial z}\left(q_{\mathrm{r}}^{n}\right)=\frac{\left(\rho_{\mathrm{c}}^{n} q_{1}^{n}\right)^{2}}{60\left(5+\frac{0.0366 C C N}{\rho_{\mathrm{c}}^{n} q_{1}^{n} m}\right)} \equiv B_{0}$.

CCN (unless given by the model, e.g., WRF-Chem) is parameterized following Rosenfeld et al. (2008) and Andreae et al. (2008) using aerosol optical thickness (AOT at $550 \mathrm{~nm}$ ):

$\mathrm{AOT}=0.0027 \mathrm{CCN}^{-0.643}$.

In WRF-Chem and/or BRAMS, AOT is provided by the simpler aerosol modules (like a bulk approach), while CCN may also come directly from the models if more complex approaches are chosen. Assuming the same unit base mass flux, we then get the rainwater conversion per base mass flux with

$\eta_{\mathrm{u}}^{n}(z) \frac{\partial}{\partial z}\left(q_{\mathrm{r}}^{n}\right)=\vartheta_{\mathrm{b}} B_{0}$,

where $\vartheta_{\mathrm{b}}$ is a proportionality factor with units of per unit mass flux. To calculate it we assume that Eq. (33) will give identical results to Eq. (30) with an average AOT value of 0.1 , which may approximate an observed global value. This means that with average conditions, Eqs. (30) and (33) will give identical rainfall conversions.

Smaller droplets will not only change the conversion from cloud water to rain water, they also may lead to an increase in evaporation. Here we follow Jiang et al. (2010) who looked at the precipitation efficiency in terms of aerosols derived from large eddy simulations of warm precipitating cumulus clouds. In their paper, they express the precipitation efficiency $P E$ in terms of the total volume of rainwater $R_{\mathrm{v}}$ accumulated at the surface and the total volume of condensed water $M_{\mathrm{v}}$ over the cloud lifetime as

$P E=\frac{R_{\mathrm{v}}}{M_{\mathrm{v}}}$.

In our parameterization $R_{\mathrm{v}}$ and $M_{\mathrm{v}}$ are normalized with the cloud base mass flux $\overline{m_{\mathrm{b}}}$, the cloud lifetime is simply the time step over which the parameterization is called. Then the precipitation efficiency, following Jiang et al. (2010) is written as

$P E \sim\left(I_{1}\right)^{\alpha_{\mathrm{s}}-1}(\mathrm{CCN})^{\zeta}=C_{\mathrm{pr}}\left(I_{1}\right)^{\alpha_{\mathrm{s}}-1}(\mathrm{CCN})^{\zeta}$, 
where $C_{\mathrm{pr}}$ is a proportionality constant that may depend on $\overline{m_{\mathrm{b}}}$, as well as the fractional coverage and that will have to be determined; $\alpha_{\mathrm{s}}$ and $\zeta$ are regression constants. We follow Jiang et al. (2010) and use $\alpha_{\mathrm{s}}=1.9$ and $\zeta=1.13$. In G1 and $\mathrm{GD}$, the precipitation efficiency $\mathrm{PE}=1-\beta$ is dependent on wind shear efficiency and sub-cloud humidity. It is a rather important parameter, since it is one of the factors that determine the strength of the parameterized downdrafts. This is even more important when considering how the proportionality factor is determined. As a simple attempt to estimate the proportionality constant, we use a similar method as was done to get the autoconversion constants. We require that under normal conditions $(\mathrm{AOT}=0.1)$ we will get the same results as if no aerosol interaction is assumed. Because of the dependence of $P E$ on $\beta$, the proportionality constant is recalculated at every grid point. If, for example, in strong wind shear and low sub-cloud humidity conditions downdrafts are already very strong and precipitation efficiency is low, an increase in CCN cannot increase the downdraft strength further, and the only change resulting from the above formulation will be a decrease in autoconversion and a resulting decrease in rainfall, as well as an increase in output of cloud water and ice (all water is assumed to be ice when the temperature is below $258 \mathrm{~K}$ ) near the cloud tops. It is also important to note here that the change in autoconversion is also considered in Eq. (35), since $I_{1}$, is depending on it. An example of the impact of these formulations on vertical heating and drying profiles are given in the next section.

\section{Applications}

As discussed in Sect. 2 of this paper, many of the assumptions that are made when parameterizing deep convection start to break down as the resolution is increased. This is of particular importance at scales where the larger-scale numerical model starts to resolve some of the convection. In this section, we present results from one-dimensional tests for Arakawa's approach (GF-A), and also the impact of the aerosol implementation on the heating and drying rates. In the second part, we will then test GF-A on three different resolutions $(20,10$, and $5 \mathrm{~km})$ and compare results with observations, simulations using $\mathrm{G} 3 \mathrm{~d}$, simulations using no convective parameterization (NO: $\mathrm{CP}$ ), and simulations using GF without any scale correction on $5 \mathrm{~km}$ resolution. Since the comparison will include some evaluation with observations we will show statistics for an average of 15 runs each.

G3d is implemented to spread the subsidence to the nearest neighbor grid points. This method has been in use in WRF for several years. It is implemented by splitting the feedback equations into two terms, lumping subsidence and massive detrainment in one term, and lateral mixing into another. The application of G3d may be envisioned as a running average as the parameterization is being applied over $3 \times 3$ grid points. The ensemble method for both G3d and GF is applied
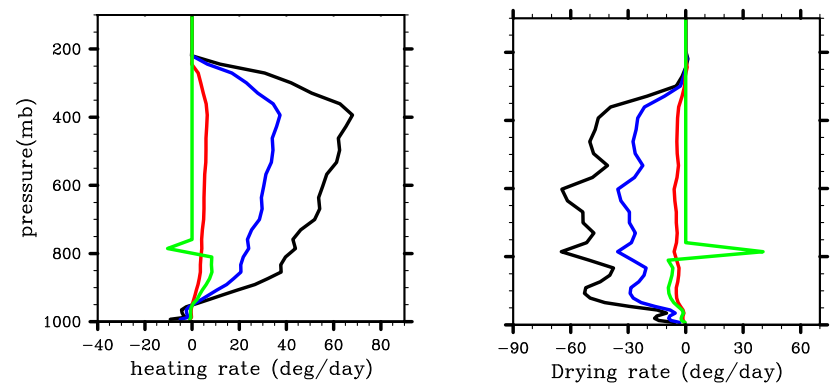

Figure 3. Heating rate (left), and drying rate (right), for grid resolution of $30 \mathrm{~km}$ (black), $10 \mathrm{~km}$ (blue), $3 \mathrm{~km}$ (red) and $1 \mathrm{~km}$ (green).

by simply feeding back the ensemble mean. Finally we will also show results for two simulations with assumed idealized clean and polluted conditions.

For the AS approach, several closures may be available for the fractional coverage of updraft and downdraft plume. For all results we are presenting below, since our intention is to keep the scheme as simple as possible, yet get a smooth transition on the gray scales, we simply use the traditional entrainment hypothesis (Simpson et al. 1965, Simpson 1971) that relates the radius of the updraft and the entrainment with

$\mu \approx \frac{0.2}{r}$.

As an additional constraint to Eq. (36), we require that $\sigma$ must be less than a given value $\tau$. Consequently the choice of the initial entrainment rate will determine when $(1-\sigma)^{2}$ becomes significant and the scale adjustment will start. We chose an initial entrainment rate to be $7 \times 10^{-5}$, which will cause significant adjustment to start at a horizontal grid-size of about $20 \mathrm{~km}$. On the other hand, another desirable outcome is that the choices of $\tau$ will affect the cloud top heights, causing a transition to precipitating shallow convection, since we force the entrainment rates to increase. Using a value of $\tau=0.25$ does not seem unreasonable and leads to significant decreases in cloud top heights for resolution of $5 \mathrm{~km}$ or better. We will present results in the next sections.

\subsection{Results using only one sounding}

For the results in this section, the convective parameterization was run offline in a one-dimensional setting. To do this we chose one grid point with active convection in a moist tropical environment from a global model simulation using NCEP's Global Forecast System (GFS). A tropical skew-t diagram and the vertical profile of moist static energy, saturation moist static energy are shown in Fig. 2. Shown also in Fig. $2 b$ is the simulated moist static energy in the updraft, assuming entrainment and detrainment ratios as given by Eqs. (20) and (36), adjusted as described above to lead to smooth normalized mass flux profiles. 

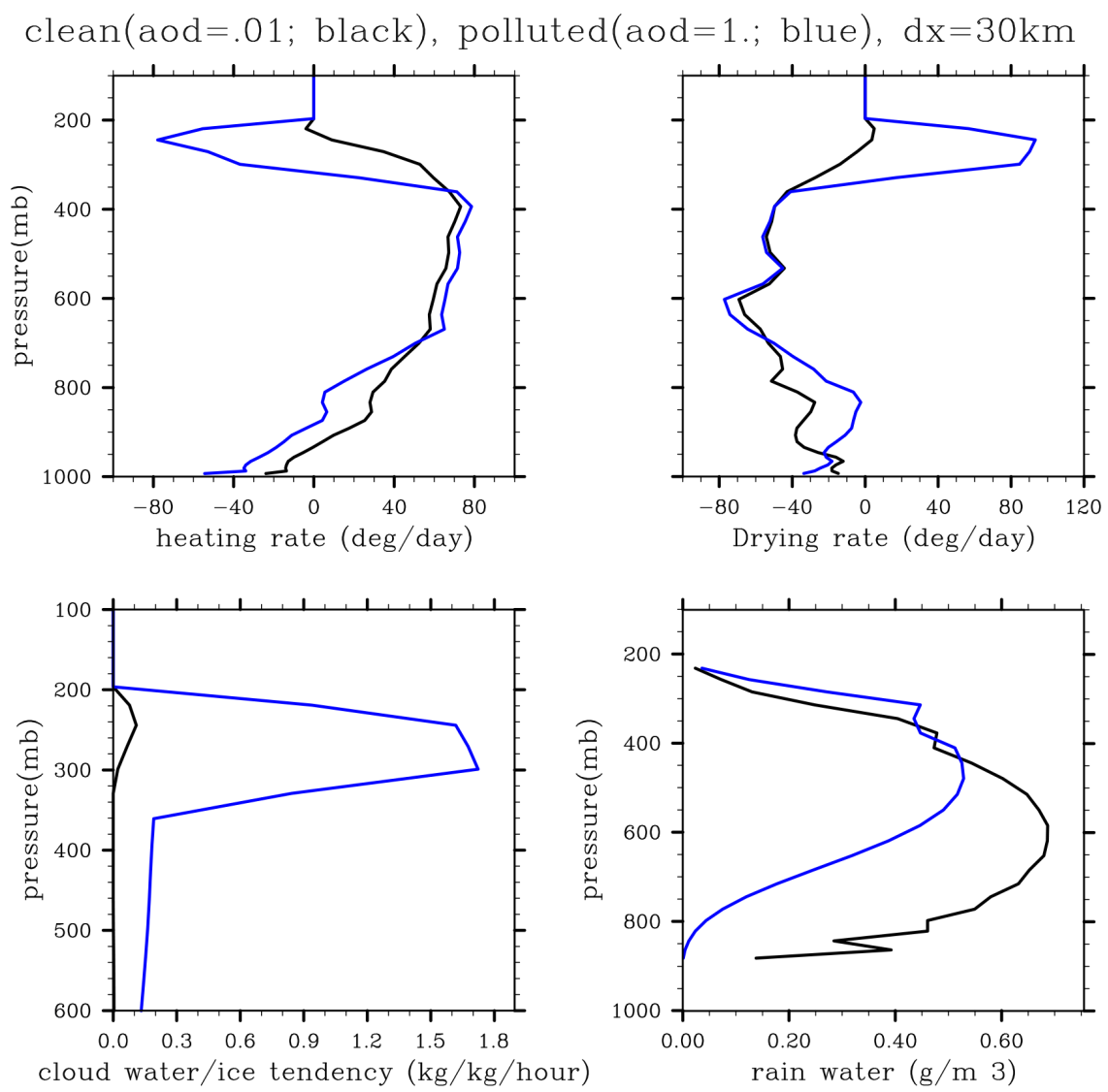

Figure 4. Vertical profiles of heating, drying, cloud water and ice tendencies, and rain water distribution for clean (black) and polluted (blue) conditions.

Temperature, moisture, and wind profiles as well as large scale forcing, were written to a file, which was then used in a simple one dimensional driver routine. In a first set of experiments, we implemented Eqs. (6) and (36), and then applied horizontal resolutions of $30 \mathrm{~km}, 10 \mathrm{~km}, 3 \mathrm{~km}$, and $1 \mathrm{~km}$ to the same sounding shown in Fig. 2. Results are shown in Fig. 3. As discussed above, with an increase in horizontal resolution the parameterizations behavior is as expected. Heating and drying tendencies decrease. In addition, for a $1 \mathrm{~km}$ resolution parameterized convection becomes much shallower the cloud top is now only at about $800 \mathrm{mb}$, down from above $300 \mathrm{mb}$.

It should be noted here that the $1 \mathrm{~km}$ resolution test in $1 \mathrm{~d}$ maybe somewhat misleading in terms of the magnitude. In 3$\mathrm{d}$ tests the convective parameterization will be automatically turned off if relative humidity is near saturation $(95 \%)$ and vertical velocity is upward anywhere below the level of free convection. For the test sounding given in Fig. 2 we have an upward vertical velocity which is explicitly used in two of the ensemble closures. In a 3-d model run the scheme would most likely be turned off for this grid box with the given sounding at high resolution, if forcing is present. We show the $1 \mathrm{~d}$ tests to see the qualitative behavior of the pa- rameterization at different resolutions without the complex interactions that happen in a numerical weather prediction Numerical Weather Prediction (NWP) model model.

Figure 4 shows the dependence on aerosol coupling for GF-A. The black curve shows results assuming a very clean atmosphere $\left(\mathrm{CCN}=50 \mathrm{~cm}^{-3}\right)$. The blue curve shows results using aerosol influence in a polluted atmosphere $\left(\mathrm{CCN}=4000 \mathrm{~cm}^{-3}\right)$. For this particular sounding heating and drying profiles when using the original $c_{0}$ approach (calculating the rainfall conversion and determining the downdraft strength without any influence of aerosols on precipitation efficiency) are almost identical compared to the run with extremely clean conditions, and are therefore not shown. This is caused since the precipitation efficiency for the control case is very high and the downdraft strength for this particular case (low wind shear, high sub-cloud humidity in the tropical environment) already is weak. On the other hand, for this environment, the polluted atmosphere has a strong influence on heating and drying rates, since downdrafts are now significantly stronger. As a consequence, low level heating and drying due to subsidence is significantly decreased (can be seen in the heating and drying profiles (blue line) in Fig. 4). Additionally, since the conversion of rainwater is much slower, 
Table 1. Summary of model runs

\begin{tabular}{clllll}
\hline Model resolution & \multicolumn{4}{c}{ Convective parameterizations } \\
\cline { 2 - 5 } & GF-A & GF-NS & G3d & NO : CP \\
\hline $20 \mathrm{~km}$ & $\mathrm{X}$ & & & \\
$10 \mathrm{~km}$ & $\mathrm{X}$ & & & \\
$5 \mathrm{~km}$ & $\mathrm{X}$ & $\mathrm{X}$ & $\mathrm{X}$ & $\mathrm{X}$ \\
\hline
\end{tabular}

rainwater is found higher up in the cloud, and much more cloud water and ice is detrained at the cloud top. The resulting rainfall tendencies are also significantly affected, with the largest rainfall amounts in the clean environment, and the least rainfall amounts in the polluted environment. It needs to be noted here that in a fully three-dimensional application non-linear effects may become much more important. Stronger downdrafts may lead to stronger convection. On scales that allow partial resolving of convection, less heating and drying may enable the numerical model to be more efficient in explicitly resolving convection.

\subsection{Three-dimensional applications}

For this study we decided to test our convective parameterizations over a South American domain using the atmospheric model BRAMS. This domain includes areas that experience organized convective systems as well as daytime local convection, which in turn may evolve into organized convective systems. To evaluate the performance of the GF schemes as well as the behavior on different scales, several experiments (GF-A) using horizontal grid-sizes of 5, 10 and $20 \mathrm{~km}$ were done. Additionally, for the runs with $5 \mathrm{~km}$ horizontal resolution we describe the performance of the scheme that spreads the subsidence (G3d), as well as a version of the scheme that does not apply any scale correction (GF-NS). Each experiment included 15 runs from 1 to 15 January for $36 \mathrm{~h}$ forecasts, all starting at 00:00 UTC. The $24 \mathrm{~h}$ precipitation accumulations used for verification are taken from 12 to $36 \mathrm{~h}$. Table 1 summarizes the different experiments.

\subsubsection{Model setup and choice of physics parameterizations}

The number of the horizontal grid points (NX, NY) were $(1360,1480),(680,740)$ and $(340,370)$ for the horizontal grid spacing of 5,10 , and $20 \mathrm{~km}$, respectively. The vertical resolution for all grids varied telescopically with higher resolution at the surface $(50 \mathrm{~m})$ up to a maximum vertical resolution of $850 \mathrm{~m}$ (a ratio of 1.1), with the top of the model at $19 \mathrm{~km}$ (a total of 45 vertical levels). The soil model was composed of 7 layers with variable resolution, distributed within the first $12 \mathrm{~m}$ of the soil depth.

For the atmospheric initial conditions, the CPTEC T213 truncation analysis fields of horizontal wind, geopotential height, air temperature, and relative humidity were used. Additionally, the CPTEC213 forecast fields, available at 6-hourly intervals, were used to provide necessary lateral boundary conditions using a nudging technique (Davies, 1983). Initial soil moisture is supplied as suggested by Gevaerd and Freitas (2006). The soil temperature was initialized assuming a vertically homogeneous field defined by the air temperature closest to the surface from the atmospheric initial data. The sea surface temperature is prescribed using the estimate developed by Reynolds et al. (2002).

Physics parameterizations include an atmospheric radiation scheme based on the Community Aerosol and Radiation Model for Atmosphere (CARMA, Toon et al., 1988, 1989; Longo et al., 2006), which accounts for interaction with hydrometeors. Surface fluxes are computed using the Joint UK Land Environment Simulator (JULES) surface scheme (Best et al., 2011), which was coupled to the BRAMS model by Moreira et al. (2013). The vertical PBL diffusion parameterization is based on the Mellor-Yamada 2.5 closure (Mellor and Yamada, 1982) formulation. For the microphysics, we used a single-moment bulk microphysics parameterization, which includes cloud water, rain, pristine ice, snow, aggregates, graupel and hail (Walko et al., 1995).

\subsubsection{Inter-comparisons of simulations using GF-A, G3d, and GF-NS}

In this section we will first describe the different behavior on the different scales and for the different cumulus parameterization options. The subsidence spreading in G3d is only turned on at $5 \mathrm{~km}$ horizontal resolution, so no model runs have been performed for G3d at coarser horizontal resolutions. Figure 5 shows the 15 day averages of total rainfall (from resolved plus parameterized convection: $\mathrm{R}+\mathrm{CP}$, upper row), and from convective parameterization (CP, lower row) in $\mathrm{mm} \mathrm{day}^{-1}$. Compared are the model results using GFA and horizontal resolutions of $20 \mathrm{~km}$ (Fig. $5 \mathrm{a}, \mathrm{d}$ ), $10 \mathrm{~km}$ (Fig. 5b, e), and $5 \mathrm{~km}$ (Fig. 5c, f). In general the predicted averaged rainfall patterns resemble the typical summer time precipitation over South America well.They are characterized by the Inter-Tropical Convergence Zone (ITCZ) and an elongated band of rainfall from the Amazon basin to the southwest of the Atlantic Ocean, called the South Atlantic Convergence Zone (SACZ). Increasing the horizontal resolution, more detailed rainfall structures are simulated, while the large-scale pattern is preserved. More importantly, as the resolution is increased, the amount of parameterized rainfall becomes less significant, with the dynamics and cloud microphysics producing a much larger fraction of the total rainfall.

In Fig. 6 we compare GF-A, G3d, and GF-NS, using a horizontal resolution of $5 \mathrm{~km}$. GF-A and G3d show similar behavior, with GF-A leading to slightly more precipitation. Additional tests (not shown here) indicated that implementing the surface flux forcing (Eqs. 21-22) in GF-A and GF-NS causes an increase in precipitation for daytime 


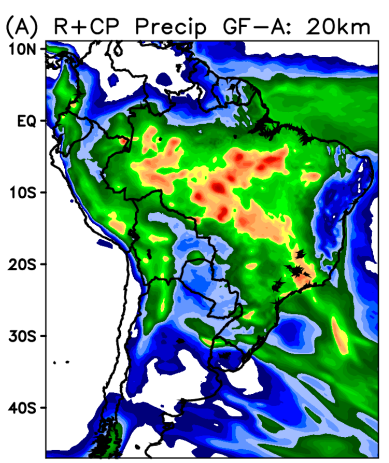

(D) CP Precip GF-A: $20 \mathrm{~km}$

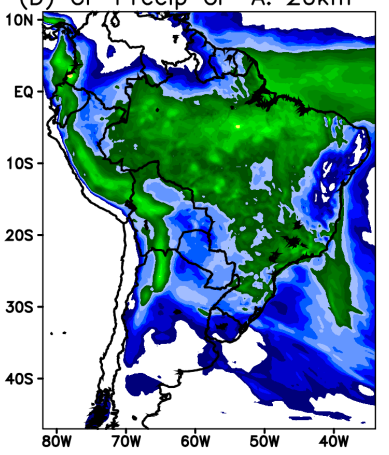

(B)

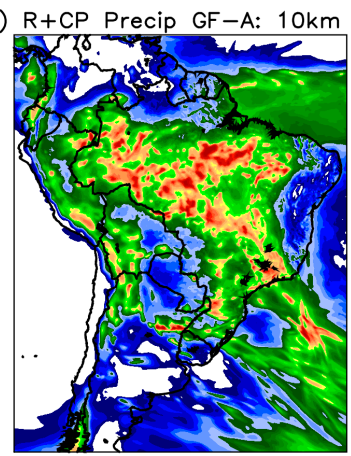

(E) CP Precip GF-A: $10 \mathrm{~km}$

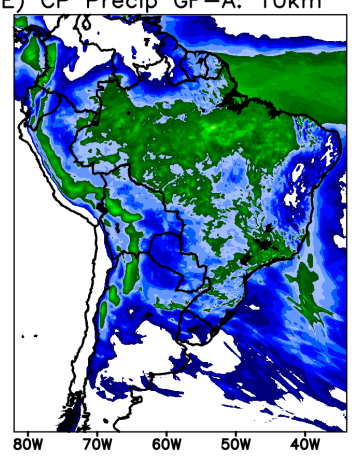

(C) R+CP Precip GF-A: 05km

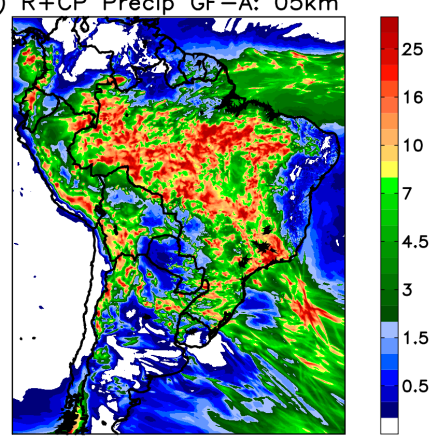

(F) CP Precip GF-A: $05 \mathrm{~km}$

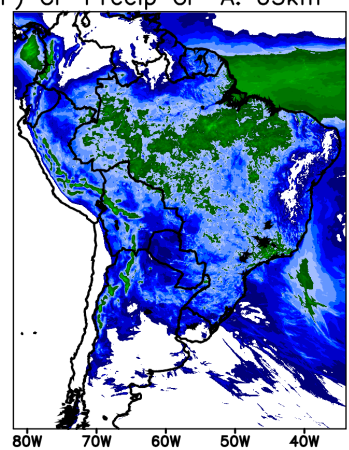

$\mathrm{mm} / \mathrm{day}$

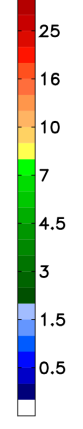

Figure 5. Averaged precipitation rates over 15 runs for total precipitation $(\mathbf{A}, \mathbf{B}$, and $\mathbf{C})$ and convective (nonresolved) precipitation rates $(\mathbf{D}$, $\mathbf{E}$, and $\mathbf{F})$, using GF-A and horizontal resolutions of $20 \mathrm{~km}(\mathbf{A}$ and $\mathbf{D}), 10 \mathrm{~km}(\mathbf{B}$ and $\mathbf{E})$ and $5 \mathrm{~km}(\mathbf{C}$ and $\mathbf{F})$. Units are $\mathrm{mm} / \mathrm{day}$.
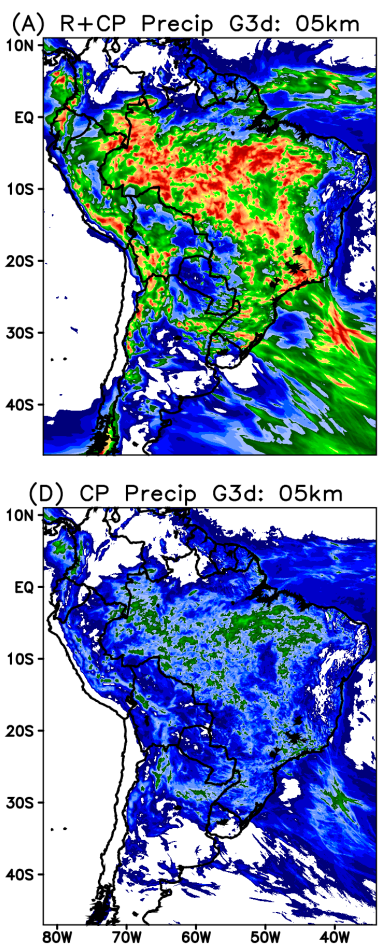

(B) $\mathrm{R}+\frac{1}{2}$

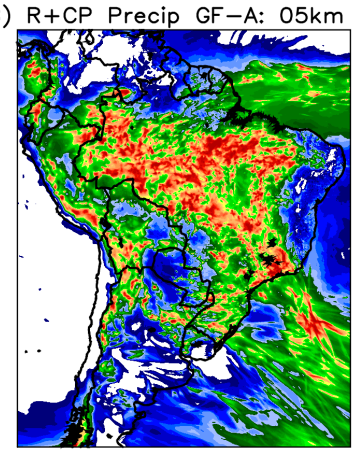

(E) CP Precip GF-A: $05 \mathrm{~km}$

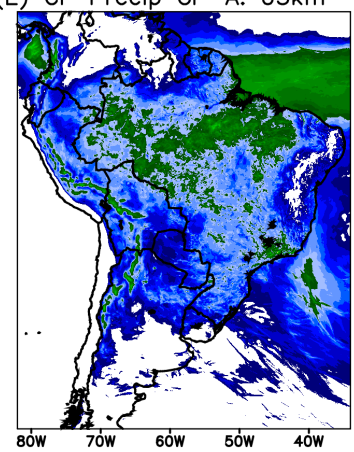

(C) $\mathrm{R}+\mathrm{CP}$ Precip GF-NS: $05 \mathrm{~km}$

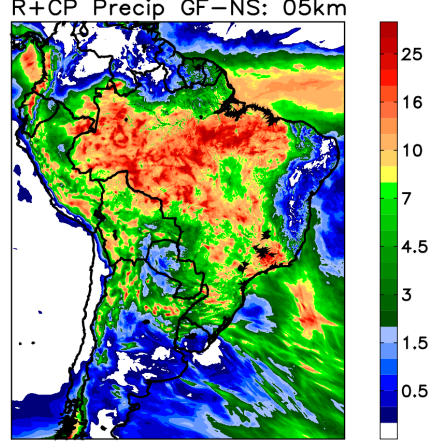

(F) CP Precip GF-NS: $05 \mathrm{~km}$

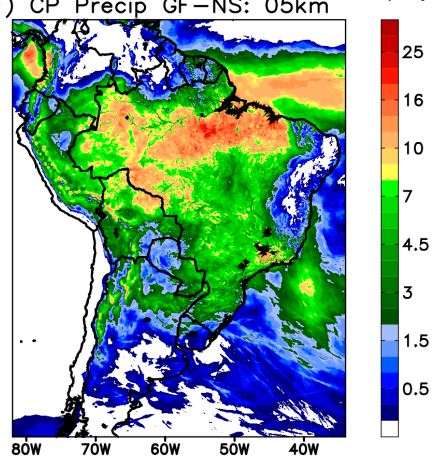

Figure 6. As in Fig. 5 except for a comparison of G3d (A and D), GF-A (B and E) and GF-NS (C and F). All use a horizontal resolution of $5 \mathrm{~km}$. 


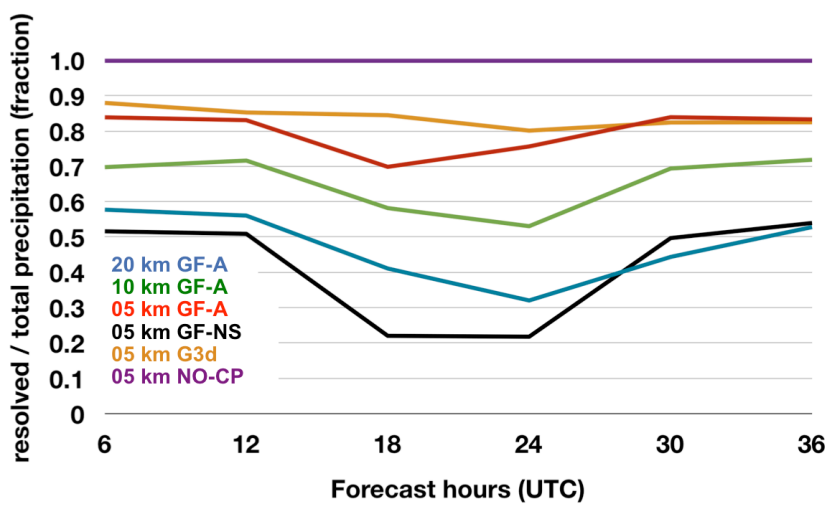

Figure 7. Fraction of resolved precipitation compared to total precipitation. 6-hourly precipitation rates are averaged for each experiment over the 15 runs and over the domain and displayed as a diurnal profile.

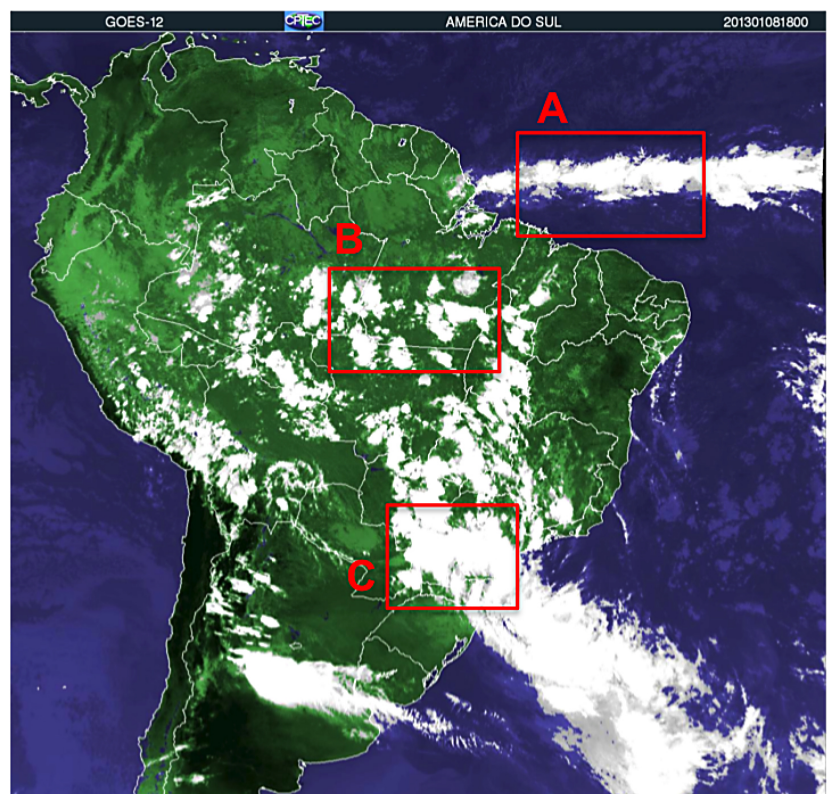

Figure 8. Satellite depiction over the domain of integration on 8 January 2013, 18:00 UTC, showing the location of three boxes A, $\mathrm{B}$, and $\mathrm{C}$ that were used for averaging.

diurnal forcing. Precipitation is also increased over the equatorial Atlantic, since the fluxes are always positive. GF-NS leads to much higher precipitation rates, especially for the non-resolved part. It should be noted here that over water, in the north-eastern part of the domain, results with GF-NS appear superior. Additionally, G3d shows significantly different behavior compared to GF-A on $5 \mathrm{~km}$ horizontal resolution. G3d and GD use many sub-ensembles, some have been weighted over water. This weighting is done in dependence of trigger functions. For GF, the stochasticism has not been explored, which is probably the reason for the difference in behavior. We are aware of this issue and are working on im-
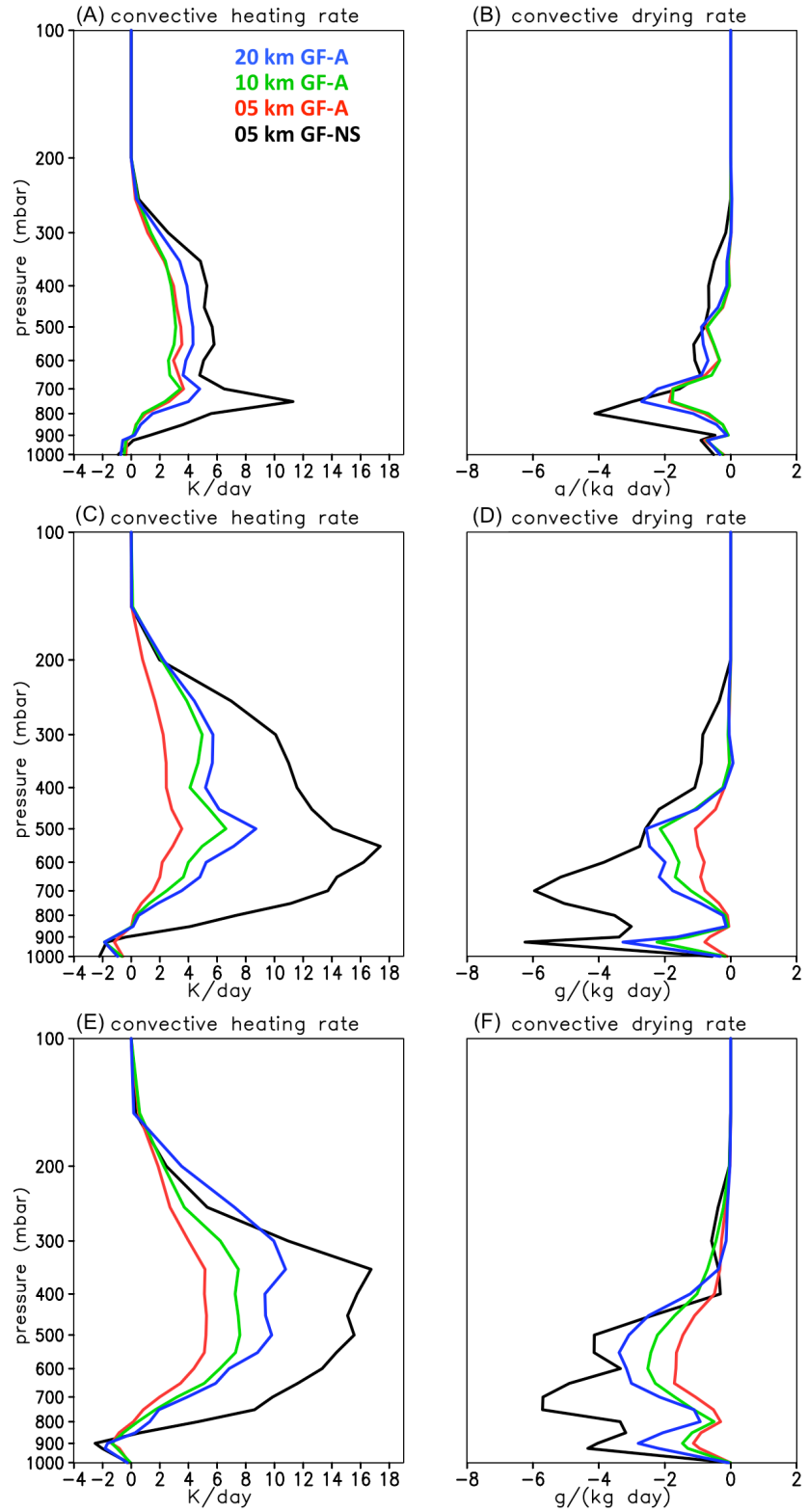

Figure 9. Vertical profiles of convective heating $(\mathbf{A}, \mathbf{C}$, and $\mathbf{E})$ and drying $(\mathbf{B}, \mathbf{D}$, and $\mathbf{F})$ for box A $(\mathbf{A}, \mathbf{B})$, box B $(\mathbf{C}, \mathbf{D})$, and box C (E, F).

plementing similar approaches into GF as were used in G3d. The fact that the total precipitation in this area is simulated best by GF-NS is not a positive outcome for GF-NS, since it would probably lead to significant errors for biases and rms errors (see also Fig. 14a and c later in the discussion), forcing all subsidence heating and drying to be in one grid box.

Figure 7 shows the diurnal evolution of the ratio between the resolved and total precipitation, spatially integrated every 6 hours. The results corroborate the discussion presented above. As expected, from 20 to $5 \mathrm{~km}$, we see a smooth transition from non-resolved to resolved precipitation as this 

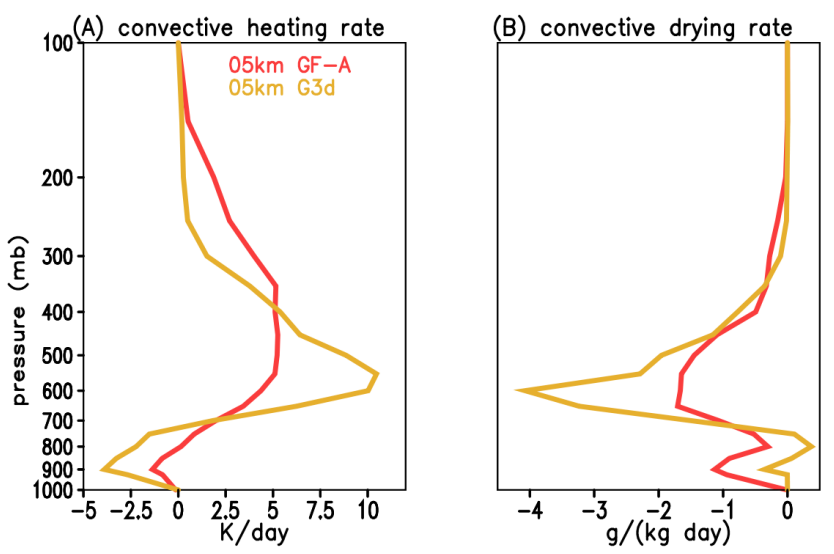

Figure 10. As in Fig. 9a and b, except for runs with GF-A on $5 \mathrm{~km}$ (red) and G3d on $5 \mathrm{~km}$ (brown) horizontal resolution.

ratio increases from $\sim 0.3-0.6$ to $\sim 0.7-0.85$. On the other hand, for GF-NS, $80 \%$ of rainfall is produced by the convective parameterization during the daytime, even on $5 \mathrm{~km}$ resolution. GF-A has a somewhat increased diurnal effect because we added the surface flux forcing. Note also that the shape of the curves for GF-A become more straight with an increase in resolution, a further indication that even for local daytime convection more of the convective precipitation is resolved. Other than the diurnal cycle effect, the ratio when using the G3d scheme is similar to GF-A on $5 \mathrm{~km}$ horizontal resolution. Obviously, the ratio for the simulation without convective parameterization $(\mathrm{NO}: \mathrm{CP}$ ) is 1 .

Next we compare averages over the areas shown as red boxes in Fig. 8 and at 18:00 UTC, 8 January 2013. First, Fig. 9 compares convective heating and drying profiles with varying assumptions and horizontal resolutions. The boxes were chosen focusing on areas that are characteristic of different convective regions over or nearby South America: the ITCZ over the equatorial Atlantic Ocean; an area over northcentral Brazil associated with daytime surface forcing and one over southern Brazil associated with a mid-latitude cold front approach. Increasing the resolution from 20 to $5 \mathrm{~km}$, the magnitude of the convective heating and drying rates decreases almost monotonically reducing the impact of convective parameterization on the model grid scale. Without Arakawa's adjustment factor, the convective heating and drying rates are much higher for GF-NS on $5 \mathrm{~km}$ horizontal resolution compared to $20 \mathrm{~km}$ resolution, a result probably related to the increased forcing for the higher resolution runs. Vertical profiles of heating and drying for both, G3d, and GF-A, are compared on $5 \mathrm{~km}$ resolution in Fig. 10 averaged over box C. They exhibit approximately similar magnitudes. In spite of averaging, results for simulations using G3d may have more vertical variability, since the normalized mass flux profiles are less smooth. There is no gradual increase of the normalized mass flux in G3d. This may also cause the somewhat stronger downdraft effects (as seen in the increased cooling and less drying in low levels).

\subsubsection{Evaluation with observations}

With Fig. 11 we begin to evaluate model performance on simulating the rainfall amount on $5 \mathrm{~km}$ horizontal grid spacing. This figure shows the spatial distribution of $24 \mathrm{~h}$ accumulated rainfall averaged over the 15 days as predicted by the various model runs and compared to observations. We also display the total domain averaged precipitation rates below each of the figure's panels over a domain bounded by longitudes $80^{\circ} \mathrm{W}$ and $30^{\circ} \mathrm{W}$ and latitudes $45^{\circ} \mathrm{S}$ and $7.5^{\circ} \mathrm{N}$. Figure 11a shows an estimate of the observed rainfall using a technique combining the Tropical Rainfall Measuring Mission (TRMM) rainfall product (Huffman et al., 2007) with the South American surface network rainfall observations (Rozante et al., 2010). The total domain averaged observed rainfall rate averaged over this domain for the 15 days was $4.38 \mathrm{~mm} \mathrm{day}^{-1}$. Figures $11 \mathrm{~b}$ and $\mathrm{c}$ show model results for GF-A and GF-NS when used at $5 \mathrm{~km}$ horizontal resolution. Results with G3d are shown on Fig. 11d, and the NO:CP case is shown in Fig. 11e. The model simulation with GFA on $5 \mathrm{~km}$ (Fig. 11b) may have a little less area coverage in the averaged precipitation distribution, and the simulated domain averaged total rainfall was $4.44 \mathrm{~mm} \mathrm{day}^{-1}$, very similar to the observed estimate. Using NO : CP or GF-NS leads to significantly higher domain averaged precipitation rates, while when using G3d the model predicts less total precipitation.

More revealing additional quantitative evaluation of the model simulations of the 6-hourly rainfall is shown in Fig. 12 using root mean square error (RMSE) and bias (mean error) calculations. The observed 6-hourly rainfall data in this case were obtained from 861 rain gauge stations distributed over South America.

For the simulations on 20,10 , and $5 \mathrm{~km}$ the daily mean values for RMSE and bias (in $\mathrm{mm} / 6 \mathrm{~h}$ ) are $(1.85,0.45)$, $(1.80,0.32)$ and $(1.81,0.12)$, respectively. G3d has similar performance with daily mean RMSE and bias of 1.83 and $0.09 \mathrm{~mm} / 6 \mathrm{~h}$, respectively. Turning off the convective parameterization completely on $5 \mathrm{~km}$, leads to a negative daily mean bias of $-0.15 \mathrm{~mm} / 6 \mathrm{~h}$ but increases the RMSE to $1.98 \mathrm{~mm} / 6 \mathrm{~h}$, larger errors than the values for the simulations using 10 and $20 \mathrm{~km}$ horizontal resolution. Using GFNS with $5 \mathrm{~km}$ horizontal resolution leads to the worst overall performance, with a considerably higher mean RMSE and bias. Also, a more pronounced diurnal cycle of RMSE and bias are seen, with the higher values during the daytime period. The best overall performance seems to be provided by the simulation on $5 \mathrm{~km}$ using GF-A and G3d.

Figure 13 shows a comparison of the commonly used equitable threat scores (ETS) and the commonly used bias scores of the 24-h accumulated rainfall for the six simulations and averaged over the 15 days. The bias score measures the ratio 
(A) TRMM+OBS Precip

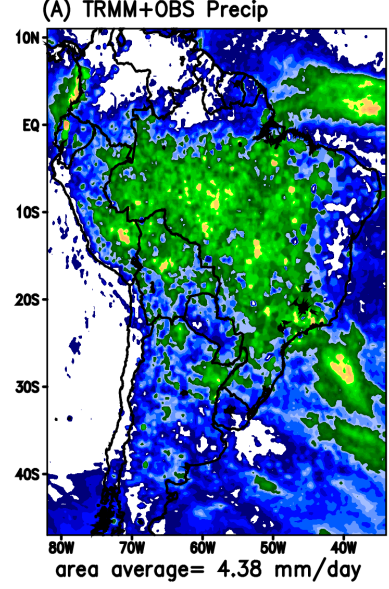

(B) R+CP Precip: GF-A 05km

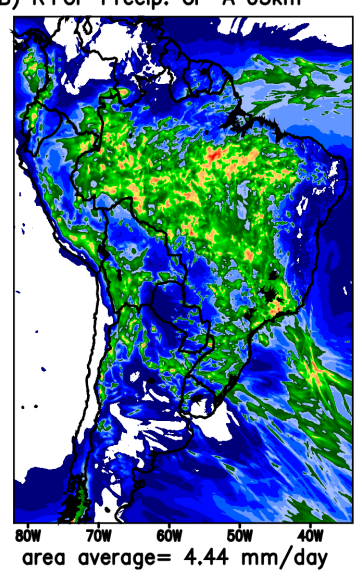

(C) R+CP Precip: GF-NS 05km

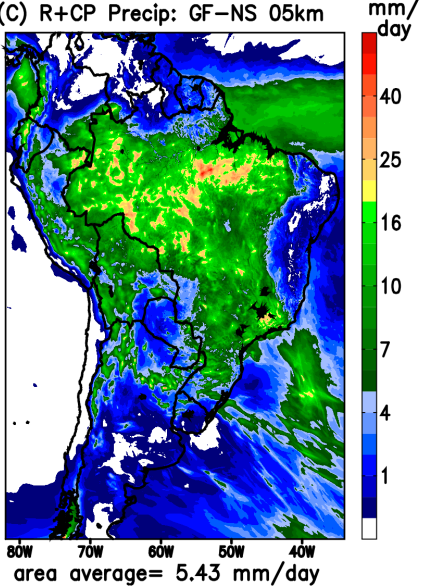

(D) R+CP Precip: $63 \mathrm{~d}$ 05km

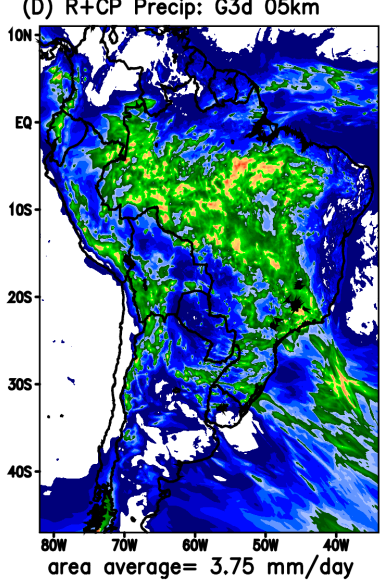

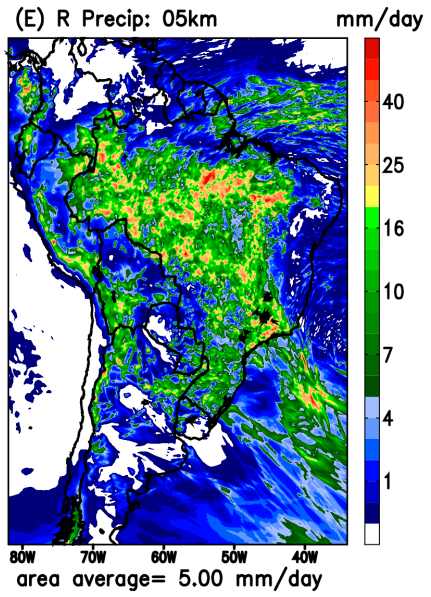

Figure 11. Comparison of averaged results using GF-A (B), G3d (D), GF-NS (C), and NO-CP (E) simulations with observations (A) derived from rain gauge and TRMM Satellite data.
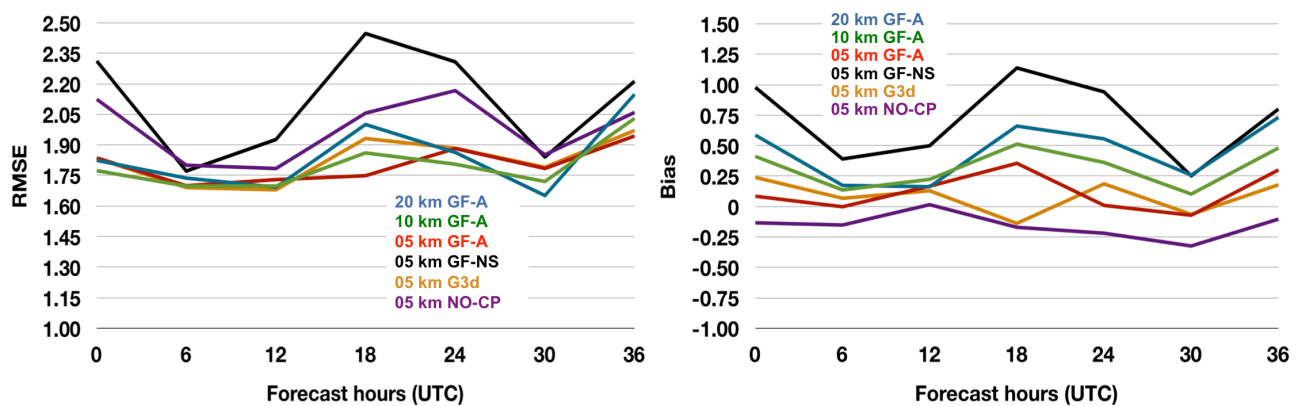

Figure 12. As in Fig. 7, except for Root Mean Square Error (RMSE) and mean error (Bias). Units are mm/6 h

of the frequency of forecast events to the frequency of observed events, binned by certain thresholds. It does not measure how well the forecast corresponds to the observations. It is also not related to the bias calculated in Fig. 12. A perfect model would obtain a value of 1 for both ETS and bias scores for any threshold.
First we notice commonly seen bias scores that are too large for all approaches for the low thresholds. Additionally, for large thresholds - there are of course less cases available - bias scores become much larger with increasing importance of resolved physics. A more detailed look reveals that for the thresholds from 0.254 to $25.4 \mathrm{~mm}$, GF-A and G3d on $5 \mathrm{~km}$ have the best bias scores, followed by GF-A on $10 \mathrm{~km}$ and 

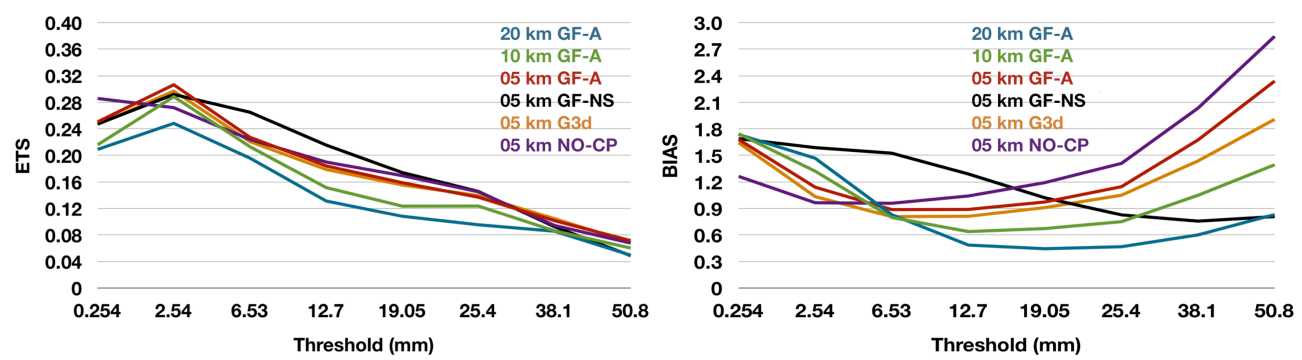

Figure 13. Equitable threat score (ETS) and bias score (bias) for the different runs averaged over the domain and displayed with respect to threshold.
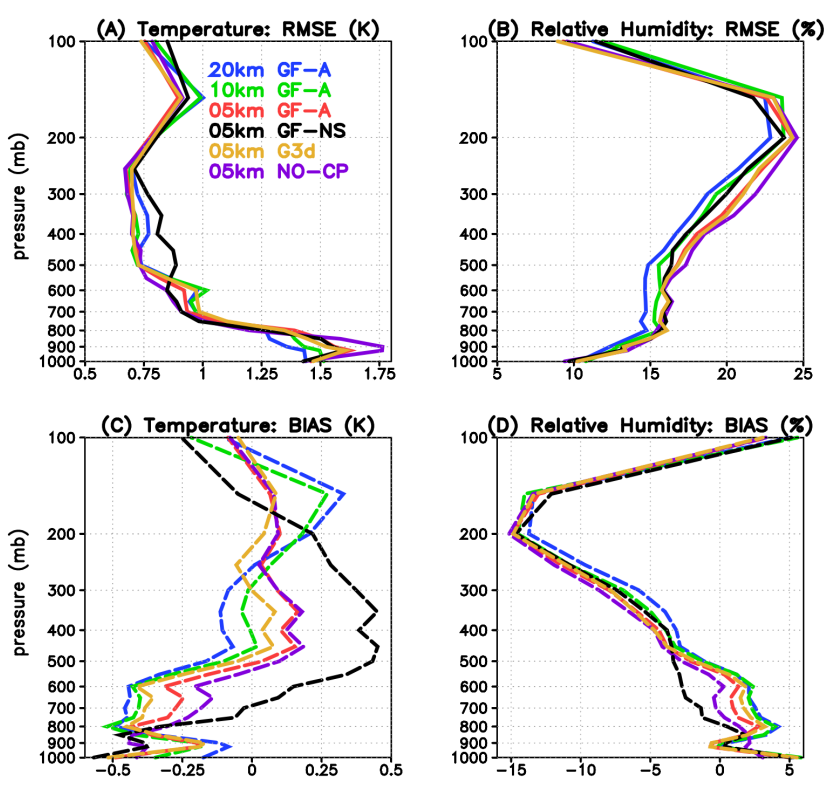

Figure 14. Vertical profiles of area averaged RMSE error $(\mathbf{A}, \mathbf{B})$ and mean error for Temperature $(\mathbf{A}, \mathbf{C})$ and relative humidity $(\mathbf{B}, \mathbf{D})$. Units are in ${ }^{\circ} \mathrm{C}$ for Temperature and percent for relative humidity.

$20 \mathrm{~km}$ resolution. For the high thresholds (above $38.1 \mathrm{~mm}$ ), coarser resolutions as well as GF-NS have better scores, but the statistical significance may be limited by the low number of cases. The number of cases for each bin are given in Table 2 .

When comparing the ETS scores, we first note that GF-NS has the highest scores for thresholds bins of 6.5 and $12.7 \mathrm{~mm}$, probably as a result of the over-forecast of events seen in the bias scores. It is not clear why the coarsest resolution GF-A runs - in spite of similar bias scores compared to GF-NS have much lower ETS scores for the very lowest thresholds. On the other hand, it is encouraging that we see an increase in ETS scores with increasing resolution. G3d and the highest resolution GF-A runs in general have very similar scores.

Figure 14 shows evaluation of the models results in terms RMSE and bias of temperature (A), and relative humidity (B), from the surface to the model top. RMSE and bias are calculated by comparing the $24 \mathrm{~h}$ model forecasts with
Table 2. Number of observations that go into calculations of bias and ETS scores.

\begin{tabular}{cc}
\hline Threshold (mm) & Number of observations \\
\hline 0.254 & 9732 \\
2.54 & 6701 \\
6.53 & 4637 \\
12.7 & 3049 \\
19.05 & 2089 \\
25.4 & 1464 \\
38.1 & 729 \\
50.8 & 382 \\
\hline
\end{tabular}

the models own initial condition, which is provided by the CPTEC global analysis. The largest improvement by far can be seen in the temperature biases, where results improve with increasing resolution and are best for GF-A, NO: $\mathrm{CP}$, and G3d. GF-NS exhibits a large warm bias over much of the troposphere, and a more significant cold bias above $200 \mathrm{mb}$. GF-A using coarser resolutions has a somewhat difference performance than GF-NS with a more significant warm bias in the upper troposphere, and not in the middle troposphere. The coarser resolution GF-A runs also exhibit a cold bias between $800 \mathrm{mb}$ and $500 \mathrm{mb}$.

RMSEs are somewhat more similar for the various experiments, except for a slight increase in RMSE for GF-NS in mid-levels, probably caused by the large temperature biases, and an increase in RMSE for the higher resolution runs - especially for $\mathrm{NO}: \mathrm{CP}$, right around $900 \mathrm{mb}$, probably caused by larger variability. This can readily happen if outflows are strong and in the wrong place.

For relative humidity (Fig. 14b), all simulations follow the same general pattern for RMSE and bias. Results are also more similar in between the different runs, except for the lower troposphere in between 800 and $500 \mathrm{mb}$. The cool bias observed in the temperature fields is replaced by a positive $\mathrm{RH}$ bias for the coarser resolution simulations. Little trust is given to the upper levels, since differences are small and analysis and observational errors may be larger. 

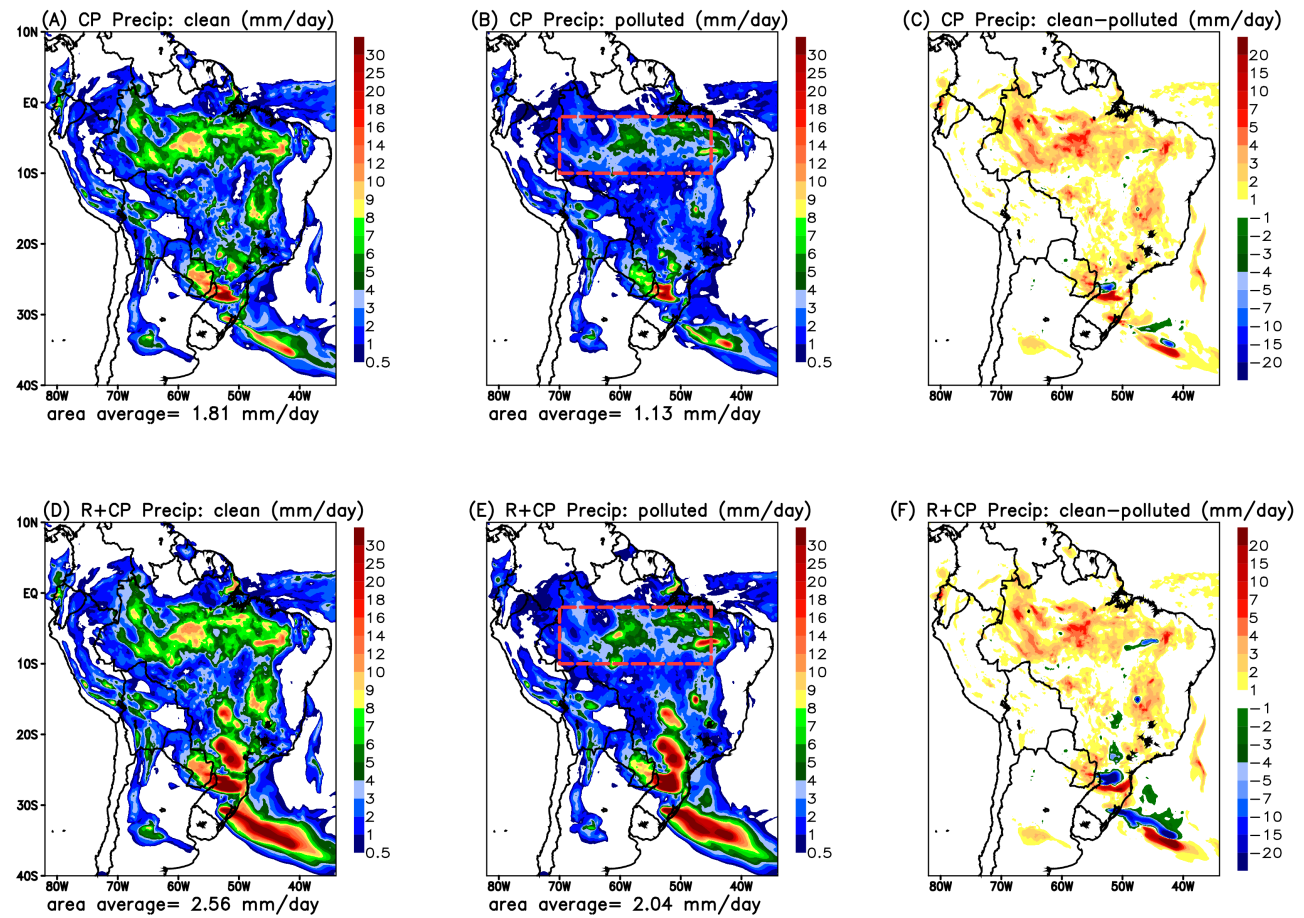

Figure 15. $24 \mathrm{~h}$ precipitation rates for a run with simulated clean conditions (A, D), polluted conditions (B, E), convective, non-resolved precipitation (A, B), the difference between clean and polluted run for convective precipitation $(\mathbf{C})$, and total accumulated precipitation (D, E) and the differences between the clean and the polluted run for the total precipitation (F).

\subsection{Aerosol interactions}

This section is dedicated to test the sensitivity of the convective parameterization to the newly implemented interaction with aerosols. We consider this implementation highly experimental, and further more detailed evaluation including a comparison to explicit WRF-Chem and BRAMS simulations is planned for the future. As an initial test, in this paper, we set up two idealized pollution conditions: clean $\left(\mathrm{CCN}=150 \mathrm{~cm}^{-3}\right)$ and polluted $\left(\mathrm{CCN}=3000 \mathrm{~cm}^{-3}\right)$. The $\mathrm{CCN}$ field is homogenously distributed in the model domain. Two simulations were conducted on a $20 \mathrm{~km}$ grid spatial resolution using the same grid and physical configurations described at Sect. 4.2. The time integration was $24 \mathrm{~h}$ starting on 00:00 UTC 8 January 2013 using the same initial and boundary conditions described before. The only difference between the two runs is the CCN field. Figure 15 shows the results related to these simulations. Figure 15a, b, and c show the $24 \mathrm{~h}$ accumulated convective precipitation for the clean (Fig. 15a) and polluted (Fig. 15b) condition, as well as the difference (Fig. 15c) between these fields The results show a reduction of the precipitation over most of the convective areas, with a domain averaged reduction of the non-resolved precipitation rate from 1.81 to $1.13 \mathrm{~mm} \mathrm{day}^{-1}$. The total precipitation on the other hand is increased in some areas (Fig. 15f), in particular in the South East part of the domain, but the whole area averaged is still reduced from 2.56 to $2.04 \mathrm{~mm} \mathrm{day}^{-1}$.
Next we focus on showing difference in heating and drying rates from the convective parameterization averaged over the red box, shown in Fig. 15b and e. This box is located over the Amazon basin; most of the simulated precipitation is of non-resolved nature. As can be seen in Fig. 15a, precipitation amounts over the $24 \mathrm{~h}$ period are decreased by almost $40 \%$. As shown earlier, the less efficient conversion from cloud water to rainwater also results in more detrainment of cloud droplets and ice, resulting in less radiation reaching the surface and a slight cooling effect near the surface (Fig. 16a). This is especially visible during the daytime, when the larger detrainment of condensate water and ice at the cloud top is causing a decrease of the domain averaged net surface radiation of up to $50 \mathrm{Wm}^{-2}$. This cooling effect may be even more enhanced because of stronger downdrafts.

Heating and drying profiles for the two runs are shown in Fig. 16b, c. Similar to the one-dimensional tests in the previous section we see two main differences. For both, the heating and drying profiles in the lower troposphere $(<4 \mathrm{~km}$ height), the larger efficiency of evaporation and the resulting increase in downdraft strength causes a decrease in the net compensating downward mass-flux, resulting in less subsidence heating and drying. Additionally, near the surface, the downdraft will detrain cool and moist (with respect to relative humidity) air. The downdraft impacts may make it easier for the microphysics to become active (especially because of the decrease in drying and heating), especially in 

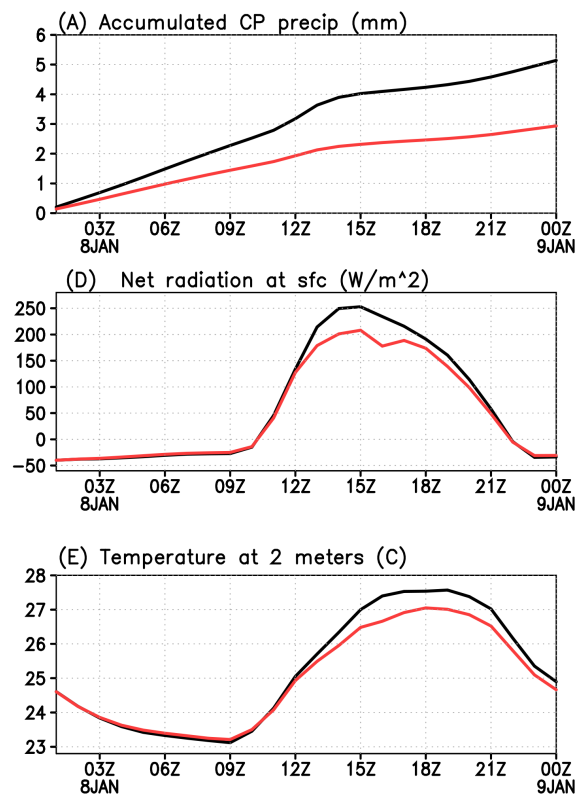
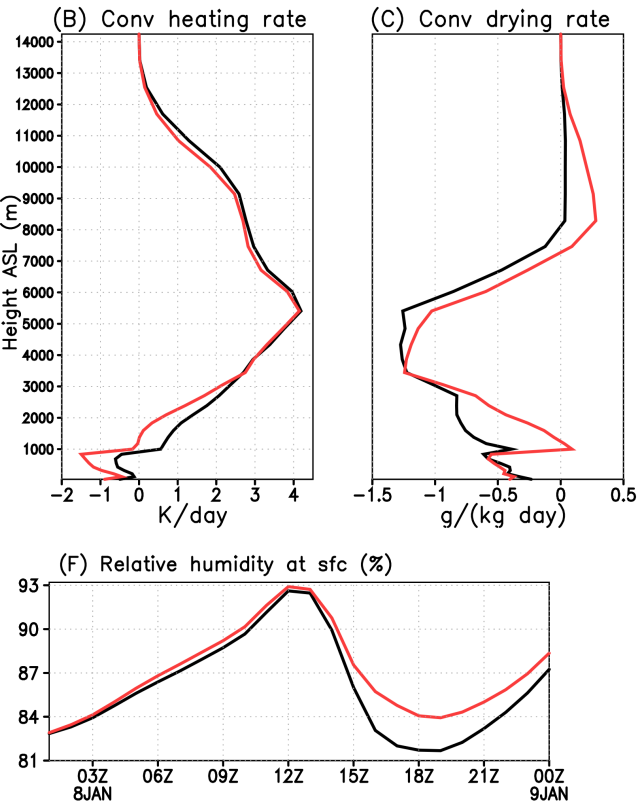

Figure 16. Various panels displaying results for clean (black) and polluted (red) conditions. Shown are accumulated precipitation (A), vertical profiles of heating (B) and drying (C) rates, net radiation at the surface (D), $2 \mathrm{~m}$ temperature (E) and relative humidity at the surface (F).

the more strongly forced mid-latitude environment with organized convective systems. A second major impact can be seen in the upper levels in the drying profiles. Above about $8 \mathrm{~km}$ the polluted runs now show a significant moistening, which is caused by the increased detrainment of condensed water and ice at and near the cloud top.

\section{Conclusions}

A convective parameterization is described and evaluated that may be used in high resolution non-hydrostatic mesoscale models as well as in modeling systems with unstructured horizontally varying grid resolutions and for convection aware simulations. This scheme is based on a stochastic approach originally implemented by Grell and Devenyi (2002). Two different approaches are tested on resolutions ranging from $20 \mathrm{~km}$ to $5 \mathrm{~km}$. One approach is based on spreading subsidence to neighboring grid points, the other one on a recently introduced method by Arakawa et al. (2011). Both approaches are available in WRF as well as BRAMS. Results using Arakawa's approach in a onedimensional application that evaluates the performance of the convective parameterization without the involvement of a complex three-dimensional model give good results with a very simple method to estimate a fractional coverage of updraft and downdraft plume. Heating and drying rates quickly become small as the resolution increases. Additionally, at resolutions of less than $3 \mathrm{~km}$, although with very small tendencies, the cloud tops become shallow. Parameterized convection is turned off completely on the highest resolutions when the air is saturated and upward vertical velocity exists. In the three-dimensional intercomparisons both the spreading of the subsidence as well as Arakawa's approach work well and give very similar results for the highest resolution runs. This also holds for a comparison to observations, where both approaches give good results. Because of its simplicity and its capability for an automatic smooth transition as the resolution is increased, Arakawa's approach is preferred. The subsidence spreading causes significant complications, since data communication is necessary. Additionally, this approach does not define a smooth transition. A smooth transition could be introduced through varying the number of grid points that are used for spreading the subsidence (or in other word the number of grid points that the parameterization is applied over), but this would complicate computational engineering even more.

Interactions with aerosols have been implemented through a CCN dependent autoconversion of cloud water to rain (Berry 1968) as well as an aerosol dependent precipitation efficiency (in combination with the existing wind shear dependent formulation of the precipitation efficiency) based on empirical results from Jiang et al. (2010). The one-dimensional comparison showed a significant increase in detrainment of cloud water and ice when using the polluted sounding (leading also to significantly less precipitation). Additionally, because of increased downdraft strength, heating and drying in the lower troposphere was much less, cooling in the lowest level increased. In a three-dimensional test we found plausible results with a decrease in predicted precipitation in some areas, probably caused by the changed autoconversion mechanism, and a significant increase of detrainment of cloud 
water and ice near the cloud tops. Some areas also experience an increase of precipitation, most likely caused by strengthened downdrafts, and as a result a more active microphysics parameterization.

Acknowledgements. The authors would like to thank John Brown for a review of the manuscript and John Osborn for editorial assistance. Additionally, two anonymous reviewers helped to significantly improve the manuscript. We would like also to acknowledge the Health of the Atmosphere (HOA) program for providing funds for this development. The second author acknowledges partial support of this work by Conselho Nacional de Desenvolvimento Científico e Tecnológico (CNPq) (306340/20119) and Fundação de Amparo à Pesquisa do Estado de São Paulo (FAPESP) (2012/13575-9). Finally we also would like to thank Graham Feingold for valuable discussions.

Edited by: J. Quaas

\section{References}

Arakawa, A., Jung, J.-H., and Wu, C.-M.: Toward unification of the multiscale modeling of the atmosphere, Atmos. Chem. Phys., 11, 3731-3742, doi:10.5194/acp-11-3731-2011, 2011.

Arakawa, A.: The cumulus parameterization problem: Past, present, and future, J. Climate, 17, 2493-2525, 2004.

Best, M. J., Pryor, M., Clark, D. B., Rooney, G. G., Essery, R .L. H., Menard, C. B., Edwards, J. M., Hendry, M. A., Porson, A., Gedney, N., Mercado, L. M., Sitch, S., Blyth, E., Boucher, O., Cox, P. M., Grimmond, C. S. B., and Harding, R. J.: The Joint UK Land Environment Simulator (JULES), model description Part 1: Energy and water fluxes, Geosci. Model Dev., 4, 677-699, doi:10.5194/gmd-4-677-2011, 2011.

Bryan, G. H., Wyngaard, J. C., and Fritsch, J. M.: Resolution Requirements for the Simulation of Deep Moist Convection, Mon. Weather Rev., 131, 2394-2416, 2003.

Davies, H. C.: Limitations of some common lateral boundary schemes used in regional NWP models, Mon. Weather Rev., 111, 1002-1012, 1983.

Emanuel, K. A.: Atmospheric Convection, Oxford University Press, New York, 580 pp., 1994.

Emanuel, K. A. and Raymond, D. J.: The Representation of Cumulus Convection in Numerical Models of the Atmosphere, Meteor. Mono., 24, Amer. Meteor. Soc., Boston, 246 pp., 1993.

Fast, J. D.; Gustafson, W. I., Easter, R. C., Zaveri, R. A., Barnard, J. C., Chapman, E. G., Grell, G. A., and Peckham, S. E.: Evolution of ozone, particulates, and aerosol direct radiative forcing in the vicinity of Houston using a fully coupled meteorology, chemistry, and aerosol model, J. Geophys. Res., 111, D21305, doi:10.1029/2005JD006721, 2006.

Frank, W. M.: The cumulus parameterization problem, Mon. Weather Rev., 111, 1859-1871, 1983.

Freitas, S. R., Longo, K. M., Silva Dias, M. A. F., Chatfield, R., Silva Dias, P., Artaxo, P., Andreae, M. O., Grell, G., Rodrigues, L. F., Fazenda, A., and Panetta, J.: The Coupled Aerosol and Tracer Transport model to the Brazilian developments on the Regional Atmospheric Modeling System (CATT-BRAMS) - Part 1:
Model description and evaluation, Atmos. Chem. Phys., 9, $2843-$ 2861, doi:10.5194/acp-9-2843-2009, 2009.

Gerard, L., Piriou, J.-M., Brozkova, R., Geleyn, J.-F., and Banciu, D.: Cloud and Precipitation Parameterization in a MesoGamma_scale Operational Weather Prediction Model, Mon. Weather Rev., 137, 3960-3977, 2009.

Gevaerd, R. and Freitas, S. R.: Estimativa operacional da umidade do solo para inicialização de modelos de previsão numérica da atmosfera. Parte I: Descrição da metodologia e validação, Rev. Bras. Meteorol., 21, 1-15, 2006.

Grabowski, W. W. and Smolarkiewicz, P. K.: CRCP: a cloud resolving convective parameterization for modeling the tropical convective atmosphere, Physica D, 133, 171-178, 1999.

Grell, G. A.: Prognostic evaluation of assumptions used by cumulus parameterizations within a generalized framework. Mon. Weather Rev. ,121, 764-787, 1993.

Grell, G. A. and Devenyi, D.: A generalized approach to parameterizing convection combining ensemble and data assimilation techniques, Geoph. Res. Let., 29, 38-1-38-4, doi:10.1029/2002GL015311, 2002.

Grell, G. A. and Baklanov, A.: Integrated Modeling For Forecasting Weather And Air Quality: A Call For Fully Coupled Approaches, doi:10.1016/j.atmosenv.2011.01.017, 2011.

Grell, G. A., Kuo, Y.-H., and Pasch, R.: Semi-prognostic tests of cumulus parameterization schemes in the middle latitudes, Mon. Weather Rev., 119, 5-31, 1991.

Grell, G. A., Peckham, S. E., Schmitz, R., McKeen, S., Frost, G., Skamarock, W. C., and Eder, B.: Fully coupled "online" chemistry within the WRF model, Atmos. Environ., 39, 6957-6975, 2005.

Hong, S.-Y. amd Dudhia, J.: Next-generation Numerical Weather Prediction, B. Am. Meteorol. Soc., 93, ES6-ES9, doi:10.1175/2011BAMS3224.1, 2012.

Huffman, G. J., Adler, R. F., Bolvin, D. T., Gu, G. J., Nelkin, E. J., Bowman, K. P., Hong, Y., Stocker, E. F., and Wolff, D. B.: The TRMM multisatellite precipitation analysis (TMPA): Quasiglobal, multiyear, combined-sensor precipitation estimates at fine scales, J. Hydrometeorol., 8, 38-55. 2007.

Jiang, H., Feingold, G., and Sorooshian, A.: Effect of Aerosol on the Susceptibility and Efficiency of Precipitation in Warm Trade Cumulus Clouds. J. Atmos. Sci., 67, 3525-3540, doi:10.1175/2010JAS3484.1, 2010.

Kessler, E.: On the distribution and continuity of water substance in atmospheric circulation. Meteorol. Mon. Amer. Meteorol. Soc., 10, 84 pp., 1969.

Khain, A. P.: Notes on state-of-the-art investigations of aerosol effects on precipitation: a critical review. Environ. Res. Lett., 4 , 015004, doi:10.1088/1748-9326/4/1/015004, 2009

Khoudier, B.: A coarse grained stochastic multi-type particle interacting model for tropical convection: Nearest neighbor interactions, Comm. Math. Sci., in press, 2014.

Kuell, V. , A. Gassmann, and A. Bott: Towards a new hybrid cumulus parameterization scheme for use in nonhydrostatic weather prediction models. Q.J.R. Meteorol. Soc.,133, 479-490, 2007.

Lin, J. W.-B. and Neelin, J. D.: Toward stochastic deep convective parameterization in general circulation models, Geophys. Res. Lett., 30, 1162, doi:10.1029/2002GL016203, 2003.

Longo, K. M., Freitas, S. R., Dias, M. A. S., Dias, and P. L. S.: Numerical modelling of the biomass-burning aerosol direct ra- 
diative effects on the thermodynamics structure of the atmosphere and convective precipitation, in: International Conference on Southern Hemisphere Meteorology and Oceanography (ICSHMO), Foz do Iguaçu. Proceedings, 8, 283-289, 2006.

Mellor, G. L. and Yamada, T.: Development of a turbulence closure model for geophysical fluid problems, Rev. Geophys. Space Phys., 20, 851-875, 1982.

Mironov, D.: Turbulence in the lower troposphere: Second-order closure and mass-flux modelling frameworks. In Interdisciplinary aspects of turbulence, edited by: Hillebrandt, W. and Kupka, F., Lect. Notes Phys., Springer-Verlag, Berlin, Heidelberg, 756, 161-221, doi:10.1007/978-3-540-78961-15, 2009.

Moreira, D. S., Freitas, S. R., Bonatti, J. P., Mercado, L. M., Rosário, N. M. É., Longo, K. M., Miller, J. B., Gloor, M., and Gatti, L. V.: Coupling between the JULES land-surface scheme and the CCATT-BRAMS atmospheric chemistry model (JULESCCATT-BRAMS1.0): applications to numerical weather forecasting and the $\mathrm{CO}_{2}$ budget in South America, Geosci. Model Dev. Discuss., 6, 453-494, doi:10.5194/gmdd-6-453-2013, 2013.

Randall, D. A., Khairoutdinov, M., Arakawa, A., and Grabowski, W.: Breaking the cloud parameterization deadlock, B. Am. Meteorol. Soc., 84, 1547-1564, 2003.

Reynolds, R. W., Rayner, N. A., Smith, T. M., Stokes, D. C., and Wang, W.: An Improved In Situ and Satellite SST Analysis for Climate, J. Climate, 15, 1609-1625, 2002.

Rozante, J. R., Moreira, D. S., De Gonçalves, L. G. G., and Vila, D. A.: Combining TRMM and Surface Observation Precipitation: Technique and Validation Over South America, Weather Forecast., 25, 885-894, doi:10.1175/2010WAF2222325.1, 2010.

Santos, A. F., Freitas, S. R., de Mattos, J. G. Z., de Campos Velho, H. F., Gan, M. A., Luz, E. F. P., and Grell, G.: Using the Firefly optimization method to weight the ensemble of rainfall forecasts of the Brazilian developments on the Regional Atmospheric Modeling System (BRAMS), Adv. Geosci., 35, 123-136, doi:10.5194/adgeo-35-123-2013, 2013.
Simpson, J., Simpson, R. H., Andrews, D. A., and Eaton, M. A.: Experimental cumulus dynamics, Rev. Geophys., 3, 387-431, 1965.

Simpson, J.: On cumulus entrainment and one-dimensional models, J. Atmos. Sci., 28, 449-455, 1971.

Skamarock, W. C., Klemp, J. B., Dudhia, J., Gill, D. O., Barker, D. M., Wang, W., and Powers, J. G.: A description of the advanced research WRF version 2, NCAR Technical Note, NCAR/TN468+STR, 8 pp., 2005.

Skamarock W. C., Klemp, J. B., Dudhia, J., Gill, D. O., Barker, D. M., Duda, M. G., Huang, X.-Y., Wang, W., and Powers, J. G.: A Description of the Advanced Research WRF Version 3, NCAR technical note, National Center for Atmospheric Research, Boulder, Colorado, USA, available at: http://www.mmm. ucar.edu/wrf/users/docs/arw_v3.pdf (7 May 2014), 2008.

Toon, O. B., Turco, R. P., Westphal, D., Malone, R., and Liu, M.: A multidimensional model for aerosols: description of computational analogs, J. Atmos. Sci., 45, 2123-2144, 1988.

Toon, O. B., McKay, C. P., Ackerman, T. P., and Santhanam, K.: Rapid Calculation of Radiative Heating Rates and Photodissociation Rates in Inhomogeneous Multiple Scattering Atmospheres, J. Geophys. Res., 94, 16287-16301, 10.1029/JD094iD13p16287, 1989.

Walko, R. L., Cotton, W. R., Meyers, M. P., and Harrington, J. Y.: New RAMS cloud microphysics parameterization part I: the single-moment scheme, Atmos. Res., 38, 29-62, 1995.

Yano, J.-I., Benard, P., Couvreux, F., and Lahellec, A.: NAM-SCA: Nonhydrostatic Anelastic Model under Segmentally-Constant Approximation. Mon. Weather Rev., 138, 1957-1974, 2010. 